\title{
Temporal subharmonic amplitude and phase behaviour in a jet shear layer: wavelet analysis and Hamiltonian formulation
}

\author{
By S. V. GORDEYEV AND F. O. THOMAS \\ Hessert Center for Aerospace Research, Department of Aerospace and Mechanical Engineering, \\ University of Notre Dame, Notre Dame, IN 46556, USA
}

(Received 11 November 1997 and in revised form 30 March 1999)

Fourier and wavelet transformation techniques are utilized in a complementary manner in order to characterize temporal aspects of the transition of a planar jet shear layer. The subharmonic is found to exhibit an interesting temporal amplitude and phase variation that has not been previously reported. This takes the form of intermittent $\pi$-shifts in subharmonic phase between two fixed phase values. These phase jumps are highly correlated with local minima of the subharmonic amplitude. In contrast, the fundamental amplitude and phase show no such behaviour. The temporal phase behaviour of the subharmonic has the effect of intermittently disrupting the phase lock with the fundamental. A dynamical systems model is developed which is based on a classic vortex representation of the shear layer. The Hamiltonian formulation of the problem is shown to provide remarkable agreement with the experimental results. All the essential aspects of the temporal amplitude and phase behaviour of the subharmonic are reproduced by the model including amplitude-dependent effects. The model is also shown to provide a dynamical systems based explanation for time-averaged amplitude and phase behaviour observed in these as well as earlier experiments. The results of experiments involving both bimodal forcing at fundamental and subharmonic frequencies with prescribed initial effective phase angle as well as experiments involving only fundamental excitation over an amplitude range extending two orders of magnitude are presented. The temporal subharmonic amplitude and phase behaviour is observed in bimodal forcing experiments in those regions of the flow characterized by subharmonic mode suppression and vortex tearing events (even if the forcing amplitudes are quite large). In addition, temporal subharmonic amplitude and phase behaviour is the rule in experiments involving low-amplitude forcing of the fundamental and the natural development of the subharmonic.

\section{Introduction and motivation}

The importance of large-scale coherent vortical structures in free shear layer dynamics is now firmly established. These structures are often described in terms of the spatial evolution of vortices, an approach which has been inspired by both flow visualization and conditional sampling experiments in jets and mixing layers. Such structures may be equivalently viewed as a superposition of interacting instability waves that amplify as they propagate in the streamwise direction. There have been attempts to bridge the gap between wave and structural descriptions. For example, Ho \& Huang (1982) showed that the streamwise locations of fundamental and subharmonic wave saturation correspond, respectively, to the average position of vortex 
roll-up and the location where pairing vortices align vertically. More recently, Yang \& Karlsson (1991) and Rajaee \& Karlsson (1992) used a superposition of the mean flow and four key instability waves (the fundamental, subharmonic, and harmonics at twice and three-halves the fundamental frequency) in order to obtain a coherent structure reconstruction of a periodically forced planar mixing layer.

Consistent with a wave description of the shear layer is the use of Fourier analysis in order to characterize transition by streamwise spectral sequences. These typically show the exponential amplification of nascent disturbances near the jet nozzle lip (or splitter plate trailing edge). Linear filtering by the shear layer leads to a well-defined fundamental instability wave whose exponential streamwise growth leads to nonlinear saturation at finite amplitude as well as the formation of multiple harmonic modes. The important role of the local subharmonic instability has been documented in numerous studies and has been shown to give rise to vortex pairing. Vortex pairing events have been found largely responsible for local increases in shear layer widening and the bulk entrainment of ambient fluid. The first vortex pairing event has also been shown to be a catalyst for the process of mixing transition which gives rise to rapid growth of small-scale turbulent fluctuations and formation of a spectral inertial subrange (Huang \& Ho 1990).

An explanation for the subharmonic mode selection in free shear layers was first provided by Kelly (1967) who performed a temporal stability analysis which indicated that when the fundamental grows to sufficient amplitude in the presence of the subharmonic wave, another instability mechanism occurs which was termed 'subharmonic resonance'. A theoretical investigation of subharmonic resonance by Monkewitz (1988) explicitly involves the nonlinear interaction between the fundamental and subharmonic under the rather strong restrictions that the flow is locally parallel and that the fundamental is a linear neutral wave. The effect of the subharmonic on the fundamental is not included in the formulation. The work establishes a critical fundamental amplitude required for resonant fundamental-subharmonic phase locking and associated enhanced subharmonic growth. The analysis predicts that at fundamental amplitude near, but less than critical, the initial deviation of subharmonic growth from exponential will be oscillatory and the amplitude will depend strongly upon the relative phase angle between the subharmonic and fundamental wave. However, this transient condition was shown to always be followed by enhanced subharmonic growth once the fundamental amplitude is above critical.

Experimental confirmation that the selective amplification of the subharmonic instability occurs by a parametric resonance with the fundamental has been made in the planar jet shear layer by Thomas \& Chu $(1991,1993 a, b)$, in the axisymmetric jet shear layer by Corke, Shakib \& Nagib (1991) and in the planar mixing layer by Hajj, Miksad \& Powers (1992). In agreement with theoretical work by Kelly (1967) and Monkewitz (1988), these experiments show that direct quadratic nonlinear energy exchange between fundamental and subharmonic is not the reason for enhanced subharmonic growth. Rather, the subharmonic is boosted due to resonant energy transfer from the mean flow associated with forced oscillation of the basic flow at the fundamental frequency (Drazin \& Reid 1983).

Arbey \& Ffowcs Williams (1984) showed that the resonant growth of the subharmonic depends on the relative phase angle between fundamental and subharmonic waves. In this paper we denote the total phase of the fundamental and subharmonic waves as $\theta_{f}=\omega_{f} t+\phi_{f}$ and $\theta_{s}=\omega_{s} t+\phi_{s}$, respectively, where the angular frequencies are related by $\omega_{f}=2 \omega_{s}$. Of interest with regard to fundamental-subharmonic phase locking is the 'effective phase angle' which is defined in such a manner as to remove 
the explicitly time-dependent portion of the total phase $\theta$. That is,

$$
\phi_{\text {eff }}=\theta_{f}-2 \theta_{s}=\phi_{f}-2 \phi_{s} .
$$

Fundamental-subharmonic phase locking is often examined within the context of which values of $\phi_{\text {eff }}$ give rise to enhanced or suppressed vortex pairing. Mankbadi $(1985,1986)$ used the energy integral technique to analytically investigate the effect of phase difference upon subharmonic growth in an axisymmetric jet shear layer. The fundamental-subharmonic $(\mathrm{F}-\mathrm{S})$ interaction was found to depend upon the effective phase angle $\phi_{\text {eff }}$. Several more recent experimental studies have applied two-frequency acoustic excitation (at fundamental and subharmonic frequencies) in order to investigate the effect of $\mathrm{F}-\mathrm{S}$ phase angle on subharmonic resonance. Yang \& Karlsson (1991) and Rajaee \& Karlsson (1992) used a phase-locked Fourier flow field reconstruction technique to show that the F-S phase of the applied excitation can either enhance or suppress vortex pairing. Pairing was observed for a wide range of initial effective phase angles but suppression of vortex pairing was restricted to a narrow range. Hajj, Miksad \& Powers (1993) applied bimodal excitation to a planar mixing layer at four effective phase angles separated by $\pi / 2$ radians. In each case the region of resonant $\mathrm{F}-\mathrm{S}$ phase locking was found to be associated with a fixed value of the local effective $\mathrm{F}-\mathrm{S}$ phase angle. With an emphasis on flow control, Husain \& Hussain (1995) examined the effect of F-S phase angle on vortex dynamics in an axisymmetric jet shear layer (with large diameter to initial momentum thickness ratio) and some of their key results are nicely summarized in figure 19 of that reference. They clearly show that vortex pairing is favoured over a wide range of effective $\mathrm{F}-\mathrm{S}$ phase angles while pairing suppression (i.e. 'vortex tearing') is maximized near a particular value of $\phi_{\text {eff }}$. This result is consistent with the analytical study of Monkewitz (1988) which suggests a clear preference for subharmonic amplification for a wide range of phase angles.

Table 1 compares the conditions for pairing or tearing examined in some of the more recent experimental efforts. In interpreting the results shown in the table, a distinction must be made between the initial effective $\mathrm{F}-\mathrm{S}$ phase angle at the jet lip or splitter plate trailing edge, $\phi_{i n}=\left.\phi_{e f f}\right|_{x=0}$, and the effective phase angle downstream at the onset of resonance. These values will not be the same. Initially the fundamental and subharmonic waves behave as independent normal modes and have different phase speeds. At sufficient fundamental amplitude, $\mathrm{F}-\mathrm{S}$ resonance commences and the effective phase angle at onset is denoted $\phi_{r}$. It is $\phi_{i n}$ that is controlled in experiments utilizing bimodal forcing and these are the values reported in table 1 (modified where necessary so as to be consistent with our definition of $\phi_{\text {eff }}=\phi_{f}-2 \phi_{s}$ ). It is clear, however, that the corresponding value of $\phi_{r}$ will depend upon the initial excitation amplitude of the fundamental and subharmonic modes, the frequency at which the excitation is applied in relation to the base flow stability characteristics and the lateral location of the measurement. Hence, the disparity in reported values of $\phi_{\text {in }}$ associated with vortex tearing is perhaps not surprising.

In the first two studies shown in table 1, detailed experiments were performed at the two reported values of $\phi_{i n}$ (although pairing was noted over a wide range of values). In the study by Hajj et al. (1993) the effective phase angle at the streamwise location of onset of fundamental-subharmonic resonance was found to be $\phi_{r}=0^{\circ}$ in each case. As indicated, Husain \& Hussain (1995) applied a much wider range of $\phi_{i n}$ and noted that most values favour pairing while vortex tearing occurs in a very limited range of phase angles.

Artificial excitation was used in each of the experiments reported in table 1 in order 


\begin{tabular}{|c|c|c|c|c|}
\hline Author & Flow † & $\begin{array}{l}\text { Excitation } \\
\text { amplitudef } \\
(\%)\end{array}$ & $\begin{array}{l}\phi_{\text {in }} \text { for tearing } \\
\text { (deg.) }\end{array}$ & $\begin{array}{l}\phi_{\text {in }} \text { for pairing } \\
\text { (deg.) }\end{array}$ \\
\hline $\begin{array}{l}\text { Yang \& Karlsson } \\
\text { (1991) }\end{array}$ & $\begin{array}{c}\text { Mixing layer } \\
R=0.33\end{array}$ & 0.4 & 275 & $75 \|$ \\
\hline $\begin{array}{l}\text { Rajaee \& Karlsson } \\
\text { (1992) }\end{array}$ & $\begin{array}{c}\text { Mixing layer } \\
R=0.32\end{array}$ & 0.4 & 270 & $90 \|$ \\
\hline $\begin{array}{l}\text { Hajj et al. } \\
\quad(1993)\end{array}$ & $\begin{array}{l}\text { Mixing layer } \\
R=0.65\end{array}$ & $\begin{array}{l}f: 0.18 \\
f / 2: 0.34\end{array}$ & 58 & $162,243,322$ \\
\hline $\begin{array}{l}\text { Husain \& Hussain } \\
\text { (1995) }\end{array}$ & $\begin{array}{l}\text { Round jet } \\
\quad \text { shear layer, } \\
R=1\end{array}$ & 0.1 & 216 & Wide range of $\phi$ \\
\hline \multicolumn{5}{|c|}{$\begin{array}{l}\dagger R=\left(U_{1}-U_{2}\right) /\left(U_{1}+U_{2}\right) \text {, where } U_{1} \text { and } U_{2} \text { are the high and low speeds, respectively. } \\
\ddagger \text { Expressed as a ratio of } U_{1} \text {. } \\
\text { I When defined as } \phi_{\text {in }}=\left.\left(\phi_{f}-2 \phi_{s}\right)\right|_{x=0} \text {. } \\
\| \text { Studied in detail at the indicated } \phi_{\text {in }} \text { but observed over a wide range. }\end{array}$} \\
\hline & \multicolumn{4}{|c|}{ TABLE 1. Experiments using bimodal excitation. } \\
\hline
\end{tabular}

to achieve 'clean' nearly spatially periodic and well-organized coherent structures that are of interest for flow control applications and/or phase-locked flow field realizations. Such artificial forcing is required to overcome the natural background perturbations, particularly at the subharmonic frequency as a result of upstream feedback. However, such forcing can give rise to behaviour that is distinctly different from that occurring in the corresponding natural flow. Flow visualization of both natural and very low-amplitude artificially excited planar shear layers actually reveals a complex sequence of events that ultimately gives rise to spatio-temporal disorder in the flow. Complications to the basic vortex pairing scenario are numerous and include spatial jitter in the vortex roll-up and pairing locations and the apparent random switching between vortex pairing and tearing events. Indeed, it is the elimination of this inherent unsteadiness of the vortical interactions that motivates the artificial forcing used in the experiments in table 1.

In order to investigate this complex, non-periodic aspect of the planar jet shear layer transition process, a continuous wavelet transform technique was applied to the hot-wire velocity time-series data, in addition to a conventional Fourier analysis. Particular attention was focused upon the temporal behaviour of the fundamental and subharmonic amplitude and phase. In so doing an interesting and previously unreported temporal behaviour of the subharmonic instability wave amplitude and phase was observed. These temporal aspects of the shear layer transition which are lost in conventional ensemble-averaged measurements and are neglected in most studies form a major focus of this work.

An objective of this paper is to focus on the temporal aspects of F-S phase locking (often characterized as phase 'jitter') as they occur in both bimodally excited and natural planar jet shear layers. We characterize a previously unreported temporal amplitude and phase behaviour of the subharmonic instability and in this regard seek to address the following questions: (i) Where and under what initial conditions does one observe the temporal subharmonic mode behaviour? (ii) How does one explain this behaviour within the framework of previous studies of shear layer transition? (iii) 
How can the observed temporal aspects of the shear layer transition be analytically modelled?

The remainder of the paper is organized as follows: In $\S 2$ the basic features of the continuous wavelet transform as it is applied in this study are discussed. In $\S 3$ the flow field facility and supporting instrumentation are described. In $\S 4$ we characterize the temporal amplitude and phase behaviour of the subharmonic that results from application of the wavelet transform. In order to relate the wavelet-based results to previous work we also present conventional measurements similar to those reported in the cited literature. This serves to: (i) establish the character of our transitioning flow field in terms of time-average amplitude and effective phase behaviour and (ii) establishes locations and initial conditions for which the jumping of subharmonic phase occurs. These experiments reported in $\S 4$ consist of two types: bimodal forcing experiments and single mode forcing experiments. The bimodal forcing experiments provide a direct bridge to the studies cited above. In the single mode experiments only the fundamental is excited acoustically at very low amplitude and for these cases the temporal behaviour of the subharmonic is commonly observed. This section investigates the natural development of the subharmonic with particular focus on the associated subharmonic amplitude and phase modulations. In combination these experiments explore the development and intermittent character of both the naturally occurring and artificially forced subharmonic and the effect upon the establishment of F-S resonant phase locking. Finally, $\S 5$ presents an analytical model inspired by the experimental results that provides understanding of the phenomenon in the context of dynamical systems theory. Numerical simulations based upon the model are also presented and these show very good agreement with the experiments. This dynamical systems model provides a very convenient context in which nearly all of the complex temporal features of the F-S interaction may be explained.

\section{The wavelet analysis of time-series data}

In this section we attempt to motivate our use of the wavelet transform and highlight some key ideas and relationships which are prerequisites for understanding the main results presented in this paper.

While Fourier analysis is capable of providing information regarding time-averaged spectral dynamics it is not well suited for characterizing the instantaneous behaviour. Because of its quasi-locality in both physical-space and Fourier-space, the wavelet transformation, which is applied in this paper, provides the capability for one to track the local time evolution of the flow. This is due to the fact that the wavelet decomposition utilizes a localized waveform as the basis function. A presentation of basic wavelet theory may be found in several recent texts on the subject (e.g. Daubechies 1992; Kaiser 1994; Farge 1992). The application of wavelet analysis techniques to experimental fluid mechanics is the topic of the paper by Lewalle (1994). In this study the temporal aspects of the transition of a planar jet shear layer are investigated by application of a Morlet wavelet decomposition to the measured streamwise, $u^{\prime}(t)$, fluctuating velocity component.

The wavelet transformation of a continuous signal $g(t)$ is defined as

$$
G_{\Psi}(\kappa, \tau)=\kappa \int_{-\infty}^{+\infty} g(t) \Psi^{*}(\kappa(t-\tau)) \mathrm{d} t
$$

where $\Psi(x)$ is the wavelet mother function, $\kappa$ is a dilatation parameter, $\tau$ is a translation or shift parameter and superscript $*$ denotes a complex conjugate. In 
equation (2.1) the wavelet transformation is written in the $L^{1}$-norm, which conserves the modulus of the function to be transformed (Farge et al. 1996). The localization of the wavelet in Fourier-physical space is governed by the dilatation parameter $\kappa$ and by the time shift $\tau$. The dilatation parameter plays a role similar to that of frequency in Fourier analysis. In other words, the scale of a given event in $g(t)$ is proportional to $1 / \kappa$. The specific relation between event scale and $\kappa$, however, will depend on the choice of the wavelet mother function. Selection of a particular mother function is based upon on what kind of information one is interested in extracting from the signal and is determined by the wavelet shape. This investigation uses a complex Morlet wavelet mother function as the basis for the wavelet transform. An analytical expression for the Morlet wavelet is

$$
\Psi(x)=\exp \left(\mathrm{i} b x-\frac{x^{2}}{2}\right)-\exp \left(-\frac{b^{2}}{2}-\frac{x^{2}}{2}\right),
$$

where $b$ is a user-specified constant. Note that since the Morlet wavelet is complex, it can provide local information concerning both amplitude and phase at a given $\kappa$. For example, if the Morlet wavelet transformation (2.1)-(2.2) is applied for the simple case of $g(t)=A \exp (\mathrm{i}(\omega t+\phi))$ (something one would not ordinarily do) where $\omega$ is angular frequency, $\phi$ is phase angle, and $A$ is a scalar amplitude, a straightforward calculation gives

$$
G_{\Psi}(\kappa, \tau)=\sqrt{2 \pi} \mathrm{e}^{-\beta}\left(1-\exp \left(-\frac{b \omega}{\kappa}\right)\right) A \mathrm{e}^{\mathrm{i}(\omega t+\phi)},
$$

where

$$
\beta=\frac{1}{2}\left(\frac{\omega}{\kappa}-b\right)^{2} .
$$

The resulting wavelet transform of $g(t)$ is a function of both time shift, $\tau$, and $\kappa$. Hence, the Morlet wavelet transform allows one to track the temporal evolution of the amplitude and phase of transient signals. Wavelet transform results are often presented in an event-duration-time space. However, in the case of existing tones with well-defined frequencies, the authors find it more convenient to present the results in a frequency-time space, $(f(\kappa), \tau)$, in order to relate them to conventional Fourier analysis. The modulus of $(2.3)$ is maximum for $\beta \approx 0$ and this allows one to relate $\kappa$ and frequency. From (2.3) we find that frequency and $\kappa$ are related by

$$
f(\kappa)=\frac{b \kappa}{2 \pi}\left(1+\mathrm{e}^{-b^{2}}+\cdots\right),
$$

which, for the value of $b=6$ used in this study, is well approximated by $f(\kappa) \approx b \kappa / 2 \pi$. Note that the wavelet transform gives only finite frequency resolution. The halfamplitude and half-power bandwidths of the wavelet transformation (2.3) are easily shown to be $\Delta f_{A} / f=2 \sqrt{2 \ln 2} / b$ and $\Delta f_{P} / f=2 \sqrt{\ln 2} / b$, respectively. They are a function of the user-specified constant $b$ and could be adjusted to any desired value. The half-amplitude bandwidth for $b=6$ is $\Delta f_{A} / f=0.39$, for example. Note that an increase in $b$, while reducing the half-amplitude or half-power bandwidths, would lead to worse resolution in the temporal domain as will be described later. The value of $b$ quoted above was found to offer the best compromise in the work to be reported.

If $\widehat{g}(\omega)$ is the Fourier transformation of $g(t)$,

$$
\widehat{g}(\omega)=\int_{-\infty}^{+\infty} g(t) \mathrm{e}^{-\mathrm{i} \omega t} \mathrm{~d} t
$$


then it is easy to show that

$$
G_{\Psi}(\kappa, \tau)=\frac{1}{2 \pi} \int_{-\infty}^{+\infty} \widehat{g}(\omega) \widehat{\Psi}^{*}\left(\frac{\omega}{\kappa}\right) \mathrm{e}^{\mathrm{i} \omega \tau} \mathrm{d} \omega .
$$

Thus an alternative and highly useful interpretation of the wavelet transformation is that at a given $\kappa$, it works like a band-pass filter in Fourier space.

Note that if plotted as a function of time the total phase term $\theta=\omega t+\phi$ obtained from the wavelet transform (2.1) will exhibit a well-known sawtooth-like oscillation behaviour (Farge et al. 1996). This behaviour appears as a consequence of the $\omega t$ term. As described in the introduction, it is the second term $\phi$ that is in fact constant for a sinusoid and that is the relevant quantity when considering $\mathrm{F}-\mathrm{S}$ phase locking through $\phi_{\text {eff }}=\phi_{f}-2 \phi_{s}$. Unless otherwise noted, in this paper the term fundamental or subharmonic phase will refer to the second term $\phi$. In particular, we will be concerned with the temporal behaviour of $\phi_{f}$ and $\phi_{s}$.

Since the focus of this work is on the temporal aspects of free shear layer transition, particularly those associated with time-dependent amplitude and phase behaviour of the subharmonic, standard Fourier analysis techniques will not suffice. Fourier analysis fails to track any temporal variation of signal amplitude and phase and could give rise to false results. In order to demonstrate this, consider a simple periodic signal which consists of the fundamental mode as a sine wave and a subharmonic mode as a cosine wave with a step-changing phase over equal increments in time:

$$
\operatorname{signal}(t)=\sin (2 \pi \times 2500 \times t)+ \begin{cases}\cos (2 \pi \times 1250 \times t+\pi / 2), & 0<t \leqslant 0.008 \\ \cos (2 \pi \times 1250 \times t-\pi / 2), & 0.008<t \leqslant 0.016 .\end{cases}
$$

Fourier transformation of this signal would give a sine wave expansion signal $(t)=$ $\sum A_{n} \sin (2 \pi n t)$ with mean subharmonic phase $\phi_{s}=0$ which is a completely misleading result, while the wavelet transformation described above would properly resolve the temporal phase variation for each mode. Figure 1 presents the results of applying the Morlet wavelet transform (2.1) to this signal. The raw signal is shown in figure 1(a) while figure $1(b)$ presents the modulus of the transform $\left|G_{\Psi}(\kappa, \tau)\right|$ in the frequency-time domain $(f(\kappa), \tau)$. The darkest regions correspond to the highest levels of the modulus. The presence of the two tones at the fundamental and subharmonic frequencies of $2.5 \mathrm{kHz}$ and $1.25 \mathrm{kHz}$ is clearly observed. Note the finite frequency resolution of wavelet transform. The time evolution of each tone can be revealed by application of (2.1) for a fixed $f(\kappa)$. For example, the amplitude variation of the subharmonic is shown in figure $1(c)$. The total phase $\theta_{s}=\omega_{s} t+\phi_{s}$ variation with time is presented in figure $1(d)$. The $\omega_{s} t$ term gives rise to the observed 'sawtooth' character which also obscures any possible variation of the $\phi_{s}$ term. However, since $\omega_{s}$ is known, it is possible to subtract the $\omega_{s} t$ term from $\theta_{s}$ and thereby investigate the time dependence of $\phi_{s}$. This result is presented in figure $1(e)$. Note that the correct subharmonic phase values of $\phi_{s}=\pi / 2$ and $\phi_{s}=-\pi / 2$ are indicated with a $\pi$-shift occurring at $t=0.008$. This figure also shows the finite time resolution of the wavelet transform as evidenced by the finite time interval over which the $\phi_{s}$ shift occurs. The fundamental amplitude and phase variations are shown in figure $1(f)$ and figure $1(g)$, respectively. Again, the wavelet transform gives the correct values for these quantities. In contrast to the subharmonic tone, the fundamental amplitude and phase are nearly constant in time.

The above example serves to illustrate that due to its filter-like behaviour, wavelet analysis can resolve the evolution of each mode independently, provided that the 
(a)

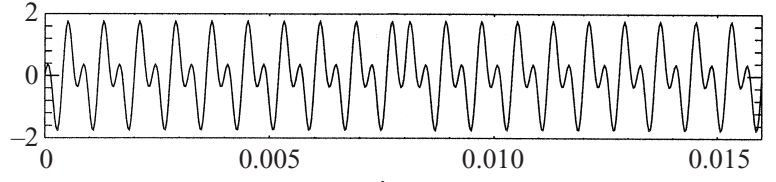

(b)

Time, $t$

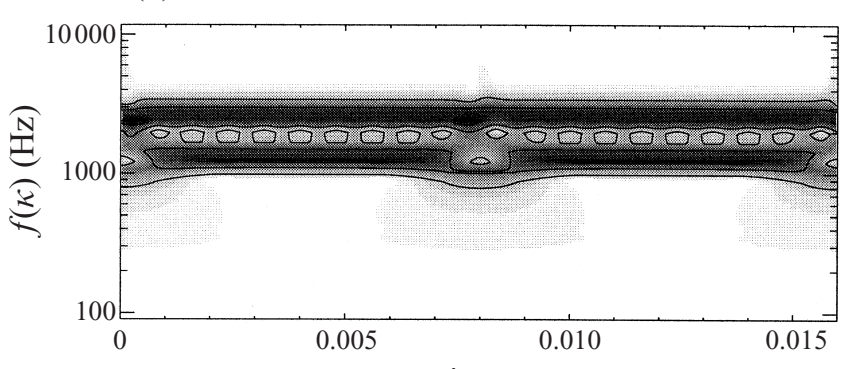

(c)

Time, $\tau$

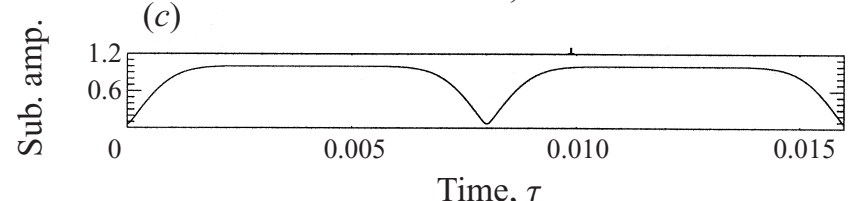

(d)

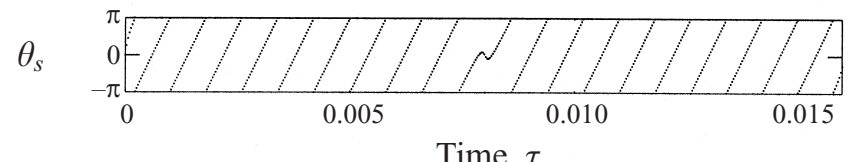

(e)
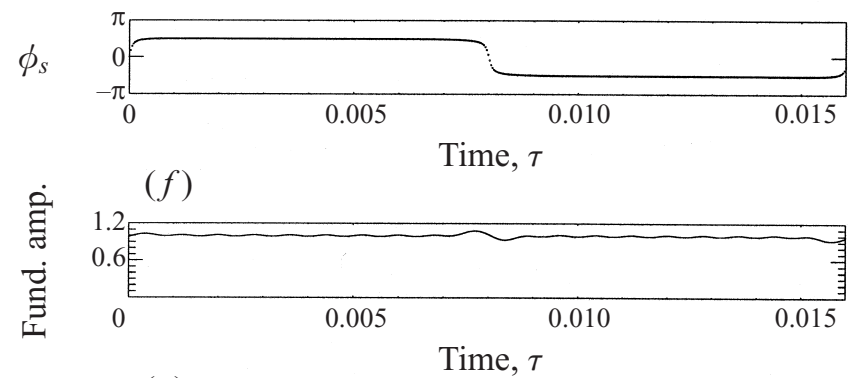

$(g)$

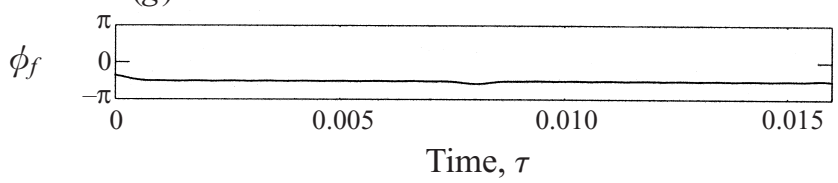

FIGURE 1. Wavelet transform of a test signal: (a) synthetic signal, defined in (2.7), $(b)$ $\left|G_{\Psi}(\kappa, \tau)\right|$ in the frequency-time domain, $(c)$ amplitude of the subharmonic mode $(f(\kappa)=1250 \mathrm{~Hz})$, (d) total phase $\theta_{s}=\omega_{s} t+\phi_{s}$ of the subharmonic mode, $(e)$ variation of the phase $\phi_{s}$ of the subharmonic mode, $(f)$ amplitude of the fundamental mode $(f(\kappa)=2500 \mathrm{~Hz}),(g)$ variation of the phase $\phi_{f}$ of the fundamental mode. 
frequency difference between modes is larger than the half-amplitude bandwidth $\Delta f_{A}$. Later this approach will be applied to the investigation of the $\phi$ phase variation of jet shear layer instability modes. This is possible since very low-amplitude acoustic excitation is applied near the most unstable shear layer frequency. Hence the fundamental and subharmonic frequencies $\omega_{f}$ and $\omega_{s}$ are known a priori.

The fundamental and subharmonic instability frequencies in the jet shear layer under investigation here occur at $2.5 \mathrm{kHz}$ and $1.25 \mathrm{kHz}$ as in the synthetic signal just considered. However, the fundamental and subharmonic bandwidths in the jet will be wider than in the synthetic signal. Further, in the real flow additional instability modes can be present that could potentially complicate application of the wavelet transform. Depending on the bandwidth of the wavelet transform at a given scale, it is certainly possible to obtain ambiguous amplitude and phase results if multiple modes are present. In the Morlet wavelet transform used in our study, the bandwidth may be adjusted through the parameter $b$ in equation (2.2). As long as the frequency spacing between spectral modes is greater than the bandwidth of the wavelet transform, the spectral peaks can be independently resolved. Jet shear layer streamwise velocity fluctuation power spectra as obtained over a wide range streamwise locations exhibit well-defined spectral peaks at the fundamental and subharmonic frequencies (Thomas \& Chu 1993a,b). Further, there are no other organized modes present within the spectral window of interest. Comparison of the measured power spectra to the halfamplitude bandwidths for the wavelet analysis corresponding to the values of $b$ used in our study indicate that this requirement is satisfied in our case. This demonstrates that the wavelet analysis can resolve the fundamental and subharmonic modes properly.

The wavelet transformation is a natural generalization of the spectral transformation concept with $\Psi(x)=\exp (\mathrm{i} x)$ a Fourier transformation. From (2.1), the wavelet transformation does not require integration over an infinite time domain centred on time $t=\tau$. Instead, integration is over a time interval proportional to $T \approx O(1 / \kappa)=$ $O(b / 2 \pi f(\kappa))$ because of a Gaussian decay of $\Psi(x)$ outside the domain. Note that $T$ is a function of the scale and thus provides a self-adjusting variable time window for different scales. This demonstrates that the wavelet transformation provides shorttime-average amplitude and phase information (on the order of $T$ ) regarding the function $g(t)$ at the moment $t=\tau$ for any unsteady signal. The price that one pays for this time localization capability is the finite frequency or scale resolution of the signal.

It should be pointed out that other techniques are available for recovering unsteady amplitude and phase modulations. A technique intimately related to the wavelet transform is the Windowed Fourier Transform (WFT). A comparative example using both the Morlet wavelet transform and WFT to analyse jet shear layer hot-wire signals will be presented in $\S 4$. In addition, the complex digital demodulation technique (Kim, Khadra \& Powers 1980) provides unsteady amplitude $A(t)$ and total phase $\theta(t)$ if the signal possesses a well-defined carrier frequency $\omega$. Use of the Hilbert transform (Bendat \& Piersol 1986) provides another option requiring only that the signal be of the form, signal $(t)=A(t) \exp (\mathrm{i} \theta(t))$ so that the temporal frequency is calculated as $f(t)=1 /(2 \pi) \mathrm{d} \theta / \mathrm{d} t$. However, each method has limitations in the sense that $a$ priori knowledge of some aspect of the signal's character is required. In contrast, the wavelet transformation provides a more general approach to investigating their transient character. Further, by the selection of different wavelet mother functions, one can highlight different aspects of the signal under investigation. That the mother function can be customized to highlight particular aspects of a signal is a real advantage of wavelet analysis. 
In practice equation (2.1) is commonly replaced by a finite discretized analogue, which is simply a numerical integral approximation for the case of a zero-order interpolation between data points:

$$
G_{\Psi}(\kappa, \tau)=\kappa \sum_{i=1}^{N} g_{i} \Psi^{*}\left(\kappa\left(t_{i}-\tau\right)\right) \Delta t_{i} .
$$

The signal $g_{i}$ is measured at discrete time points $t_{i}, i=1 \ldots N$ with $\Delta t_{i}=t_{i+1}-t_{i}=$ $\Delta t=$ const. Higher-order interpolation schemes can be used to improve accuracy. The theory of frames bounds the errors of the discrete wavelet calculations (Daubechies 1992; Kaiser 1994) which can be large for very coarse discretization. We find that in our case this computational error never exceeds $0.1 \%$. In particular, for the Morlet wavelet it follows from investigation of equation (2.6) that the maximum resolution is $\kappa_{\max }=\pi /((b+3) \Delta t)$ so that the maximum correctly resolved frequency is $f_{\max }=b /((b+3) \Delta t)$. Consequently, in the case of the Morlet wavelet, the sampling frequency should be at least $(2(b+3)) / b$ times higher than the maximum frequency to be resolved. Obviously, $\kappa_{\min }$ is on the order of $1 / T$, where $T$ is the total sampling time.

\section{The flow field facility}

The experiments were performed in the developing shear layer of a planar jet flow field facility at the Hessert Center for Aerospace Research located at the University of Notre Dame. This is the same facility described in Thomas \& Chu $(1993 a, b)$ and therefore only a brief description of essential flow parameters will be presented here.

The two-dimensional nozzle is based upon a cubic contour with zero-derivative end conditions and has a contraction ratio of $16: 1$, ending in a slot exit that is $D=1.27 \mathrm{~cm}$ in width and $H=45.7 \mathrm{~cm}$ in height (i.e. aspect ratio $=36$ ). A duct connecting the plenum chamber to the nozzle assembly contains acoustic baffling, honeycomb flow straighteners and multiple turbulence reduction screens. The measurements were performed at a Reynolds number (based upon exit mean velocity, $U_{0}$, and nozzle slot width, $D$ ) of approximately $R e_{D}=1.7 \times 10^{4}$ which corresponds to an exit velocity of $U_{0}=20.7 \mathrm{~m} \mathrm{~s}^{-1}$. The exit longitudinal turbulence intensity as measured on the jet centreline is less than $0.04 \%$. The jet initial mean velocity profiles are flat (i.e. 'top-hat' shape) and the mean velocity variation across the nascent jet shear layers is closely approximated by a hyperbolic tangent type of profile. The free shear layers at the nozzle lip are both laminar and have an initial momentum thickness $\theta_{0}=0.14 \mathrm{~mm}$.

In order to facilitate control of the initial instability in frequency and amplitude, a loudspeaker was mounted in the duct upstream of the nozzle assembly. Two types of excitation were used. The first involved bimodal forcing at the fundamental and subharmonic frequencies in which case the signal to the loudspeaker was of form $A_{f} \cos \left(2 \pi f_{e} t\right)+A_{s} \cos \left(\pi f_{e} t-\phi_{i n} / 2\right)$. In this manner the exit perturbation amplitudes of the fundamental and subharmonic could be individually controlled as was the initial effective phase angle, $\phi_{i n}$. The fundamental excitation frequency was always $f_{e}=$ $2.5 \mathrm{kHz}$ which is very near the most unstable shear layer frequency and corresponds to a Strouhal number based on exit momentum thickness of $S t_{\theta}=0.017$. Measurements made with bimodal forcing allow comparison with results from works cited in the introduction and also provide the framework for a main focus of this study which is the temporal aspects of the naturally occurring subharmonic.

In the second type of experiment only the fundamental instability was artificially 
excited. No attempt was made to force the subharmonic instability, which developed naturally. For these experiments, the exit fluctuation intensity within a narrow frequency band centred on the fundamental frequency ranged from high to very low levels: $0.002 \% \leqslant\left(\overline{u^{2}}\left(f_{e}\right)\right)^{1 / 2} / U_{0} \leqslant 0.1 \%$. Note that this range extends two orders of magnitude lower than the forcing levels used in the studies listed in table 1. For the lowest excitation amplitudes, the initial disturbance level was extremely small and the shear layer inflectional instability mechanism provided 'natural' streamwise amplification.

Constant-temperature anemometers were used in conjunction with conventional str aight wire probes to acquire the $u^{\prime}(t)$ time series signals required for the Fourier and wavelet analysis. A laboratory $\mathrm{PC}$ was used for the digital data acquisition and for traverse system control. The digital time-series data was sampled at $10 \mathrm{kHz}$ and was off-loaded to a Sun Sparc 10 station for post processing.

In this paper $x$ denotes the streamwise spatial coordinate measured from the nozzle exit plane while $y$ is the cross-stream spatial coordinate whose origin is located at the centre of the jet shear layer (i.e. $U(y) / U_{0}=0.5$ ). Unless otherwise noted, the lateral measurement location corresponds to a position in the shear layer for which the local subharmonic wave amplitude is maximum. The measurements were performed at streamwise $x$ locations extending from the jet lip to downstream of subharmonic saturation (i.e. well upstream of the tip of jet potential core).

\section{Experimental results}

When the continuous Morlet wavelet transform as described in $\S 2$ was applied to streamwise velocity fluctuation time series in the transitioning planar jet shear layer, an interesting and previously unreported temporal behaviour of the natural subharmonic instability was observed that we next describe. By the term 'natural subharmonic' we imply that only the fundamental instability was artificially excited at very low amplitude $\left(\overline{u^{2}}\left(f_{e}\right)\right)^{1 / 2} / U_{0} \equiv u_{f}^{\prime}=0.01 \%$ with no artificial excitation of the subharmonic instability used so that it develops naturally in the flow. Deferring presentation of details pertinent to the measurement to later in $\S 4$, for now we simply refer the reader to figure 2 for the purpose of illustrating a representative sample of this temporal subharmonic behaviour as derived from the wavelet analysis. This figure presents the time evolution of subharmonic amplitude, subharmonic phase $\phi_{s}$, and the effective phase $\phi_{\text {eff }}$, and shows the existence of two steady values of subharmonic phase $\phi_{s}$ with intermittent $\pi$-jumps between them. Note that these jumps are well correlated with local drops in the subharmonic amplitude. This aspect is highlighted in figure 2 for one of the subharmonic phase-shift events. In contrast, time intervals of stable subharmonic phase are associated with elevated subharmonic amplitude. This behaviour is unique to the subharmonic instability; the fundamental exhibited comparatively stable amplitude and phase.

As noted earlier, a technique that is closely related to the wavelet transform is the Windowed Fourier Transform (WFT). However, the WFT provides only average values of amplitude and phase of the mode over the data block size. Furthermore the block size is fixed for all frequencies and the averaging over it leads to the possibility of concealing temporal variations of the amplitude and phase that occur on time scales shorter than the block size. Obviously what one sees will then depend on the window length and proper adjustment of the block size can require considerable prior knowledge of the signal. In contrast, in $\S 2$ it was shown that the wavelet transform utilizes a variable time-window approach, which allows one to recover the dynamics 

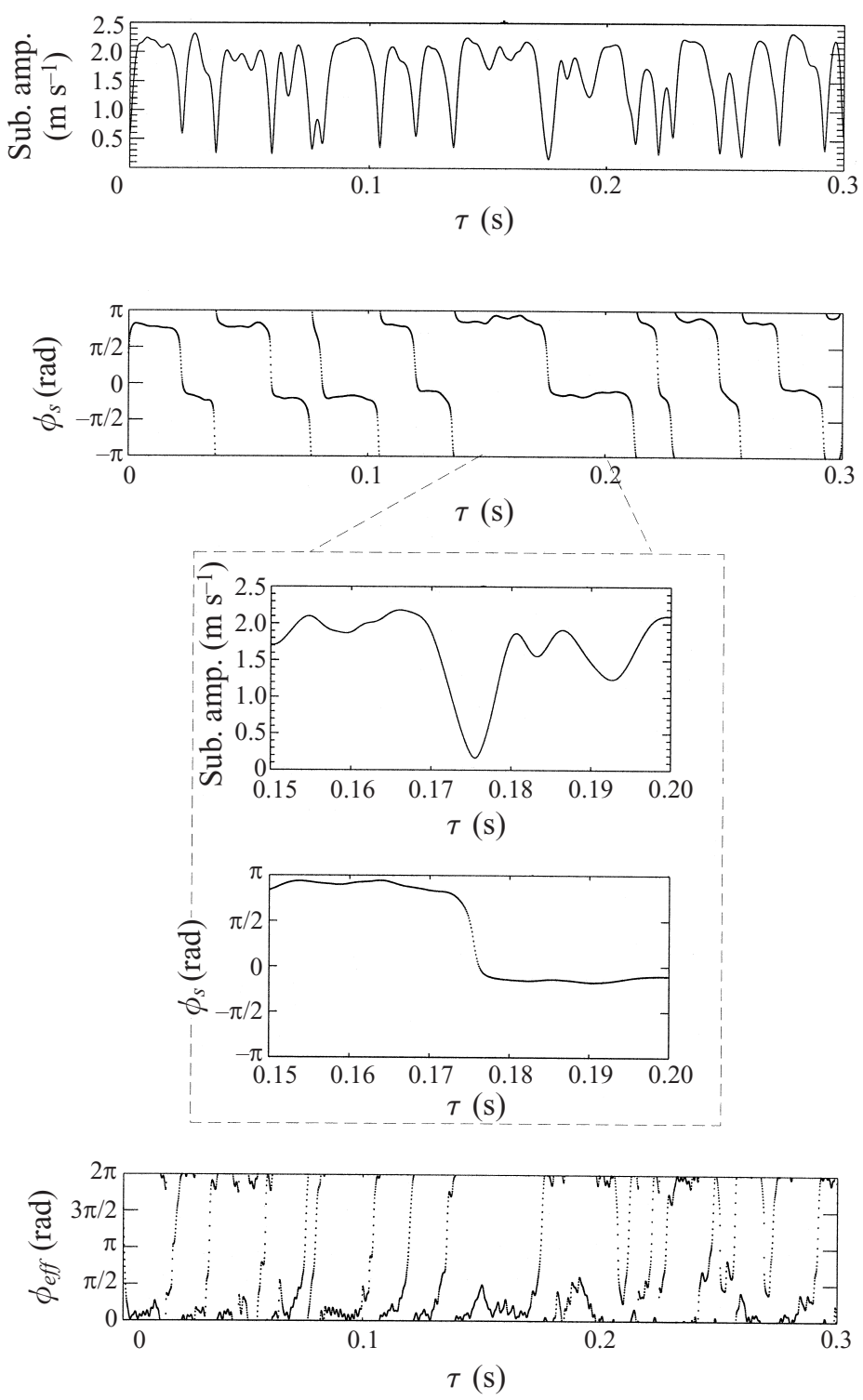

FIGURE 2. Temporal variation of subharmonic amplitude, phase and effective phase: $u_{f}^{\prime}=0.002 \%$, $u_{s}^{\prime}=0, x / \theta_{0}=110$.

of the signal at any frequency with minimal prior knowledge about the signal one has properly sampled. For example the temporal amplitude and phase behaviour of the subharmonic mode shown in figure 2 was found by direct application of the Morlet wavelet transform. However as shown in figure 3, with prior knowledge of the time scales involved in the dynamics as gleaned from wavelet analysis, the WFT is capable of showing the same essential characteristics, albeit less clearly. Actually, these drawbacks of the WFT led to the development of the wavelet transform. An excellent discussion highlighting both the similarities and key differences about the WFT and the continuous wavelet transform can be found in $\S \S 2$ and 3, respectively 

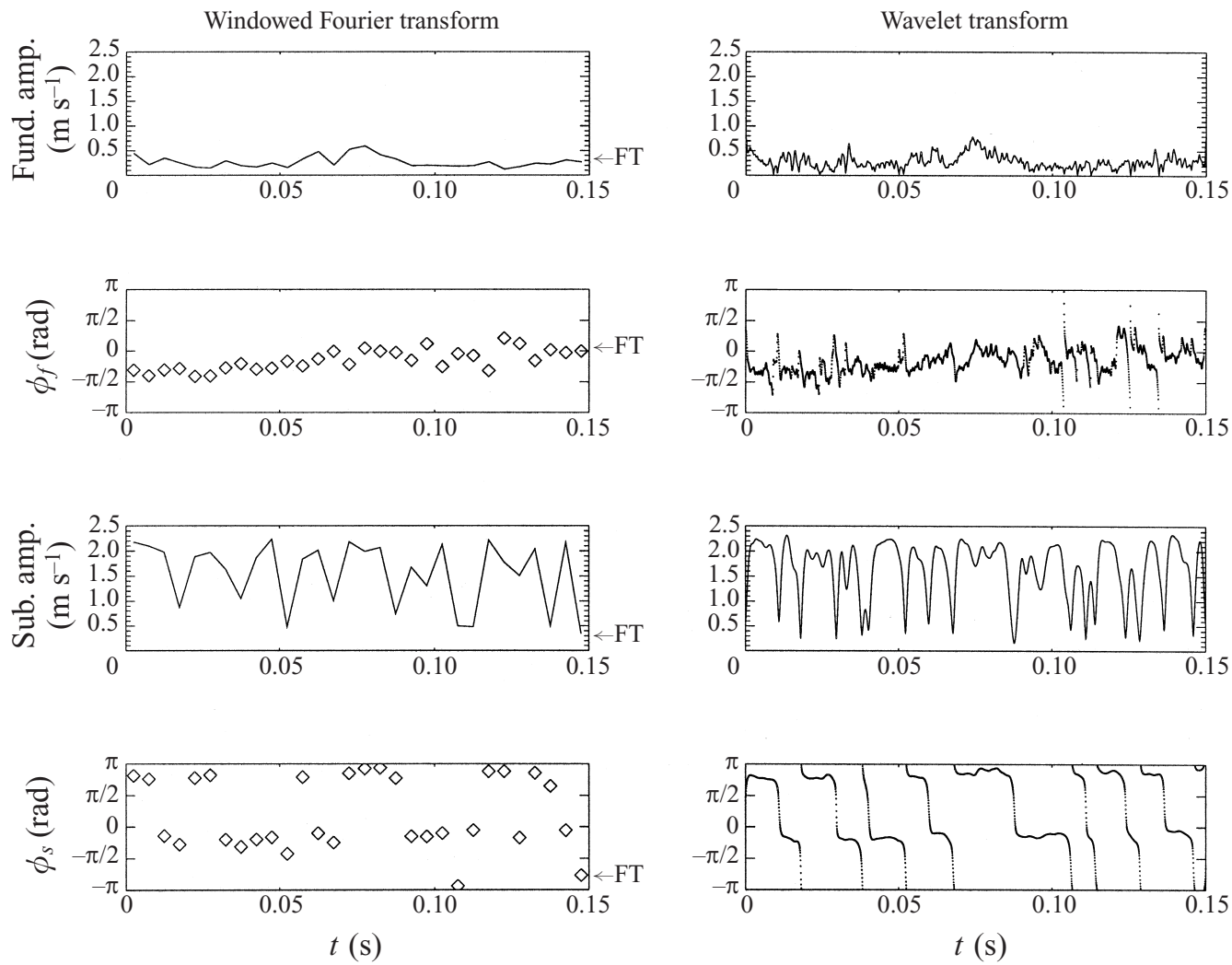

FiguRE 3. Comparison between windowed Fourier and wavelet transforms.

of Kaiser (1994). Also shown in figure 3 are the values of subharmonic amplitude and phase provided by conventional Fourier transform analysis (designated by FT).

A systematic series of experiments was undertaken in an effort to (i) determine the locations and initial conditions for which temporal subharmonic behaviour like that shown in figure 2 occurs, (ii) relate these effects to the previously cited studies which use conventional Fourier analysis techniques. In the following portions of this section results from two types of experiments are presented. Consideration is first given to the case of bimodal forcing at the fundamental and subharmonic frequencies over a wide range of initial $\mathrm{F}-\mathrm{S}$ phase angles. The forcing amplitudes for these experiments are similar to those used in the investigations reviewed in $\S 1$. The purpose of these experiments is to provide a basis for comparison with previous work and to set the framework for contrasting with results involving the natural subharmonic shear layer evolution. For these measurements we utilize experimental methods similar to those used in the cited literature in addition to the wavelet analysis technique.

It is the natural subharmonic evolution that forms the focus of the second group of experiments. For these, only the fundamental instability is excited at low amplitude while the subharmonic is allowed to develop naturally. The shear layer dynamics observed in these experiments are characterized by an inherent temporal variation that forms a major focus of this work. The observed temporal behaviour of the subharmonic instability is the motivation for a Hamiltonian system approach to vortex pairing and tearing interactions in the shear layer. However, it will be later 

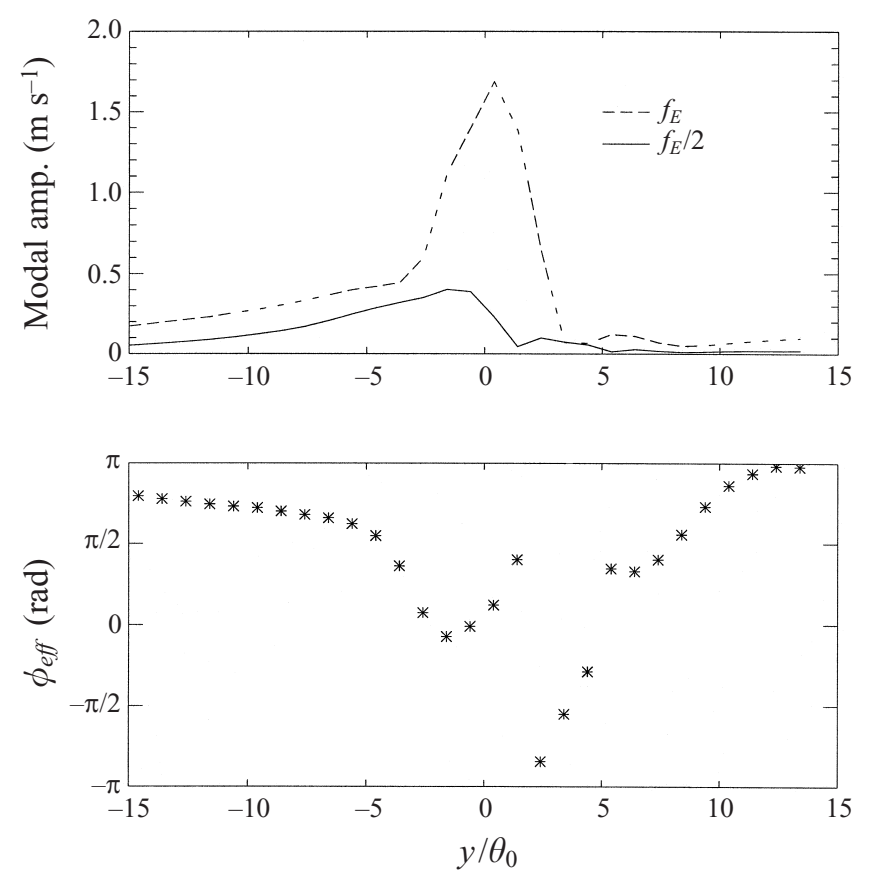

FIGURE 4. Measured cross-stream variation of fundamental and subharmonic amplitudes and local effective phase at $x / \theta_{0}=60$ for $\phi_{i n}=180^{\circ}$.

shown that the resulting model also predicts many aspects observed in the experiments using bimodal forcing at higher amplitude.

Measurements were performed at several lateral locations within the jet shear layer. However, since a focus of our paper is on the dynamic behaviour of the subharmonic instability, the data that we present below were obtained near the location of maximum subharmonic amplitude. These may be considered representative of results obtained at the other lateral locations, which exhibited qualitatively similar dynamic behaviour.

\subsection{Bimodal forcing experiments}

In experiments using bimodal forcing at fundamental and subharmonic frequencies, several amplitude combinations were explored. In this section we will present results obtained for two of these combinations that are representative of the type of behaviour encountered and which serve to provide a basis for comparison with previous studies. In the first case $\left(\overline{u^{2}}\left(f_{e}\right)\right)^{1 / 2} / U_{0} \equiv u_{f}^{\prime}=0.01 \%$ and $\left(\overline{u^{2}}\left(f_{e} / 2\right)\right)^{1 / 2} / U_{0} \equiv u_{s}^{\prime}=0.1 \%$. In the second case both fundamental and subharmonic forcing amplitudes were the same: $u_{f}^{\prime}=u_{s}^{\prime}=0.1 \%$. The fairly high level of excitation used in these experiments modifies the shear layer dynamics in the sense that it reduces the natural 'phase jitter' and allows the use of standard Fourier analysis techniques for investigating the spatial amplitude and phase behaviour of the developing modes.

In terms of tracking the streamwise evolution of the effective $\mathrm{F}-\mathrm{S}$ phase angle care must be taken in the selection of the lateral location of the measurement. As an example, figure 4 shows a typical cross-stream profile of the fundamental and subharmonic modal amplitudes as well as the local effective $\mathrm{F}-\mathrm{S}$ phase angle $\phi_{\text {eff }}$ obtained at the $x / \theta_{0}=60$ location for the initial effective phase $\phi_{\text {in }}=180^{\circ}$. It is apparent from this figure (and other cross-stream profiles that were obtained but are 
(a)

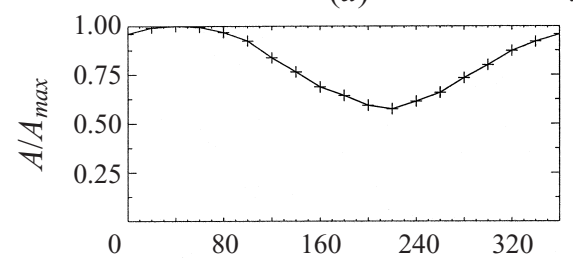

$x / \theta_{0}=50$

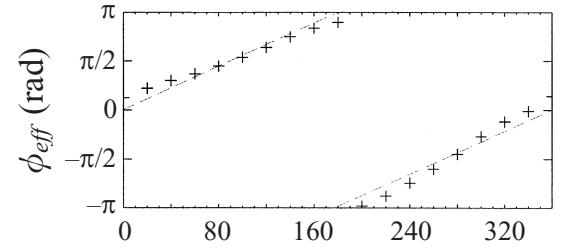

$x / \theta_{0}=60$
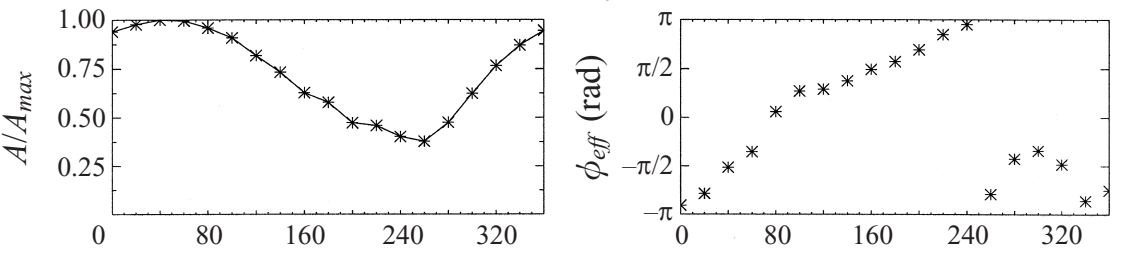

$x / \theta_{0}=80$

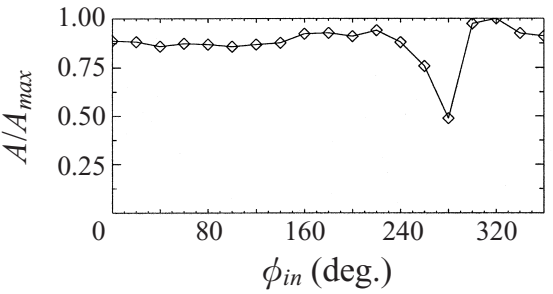

(b)

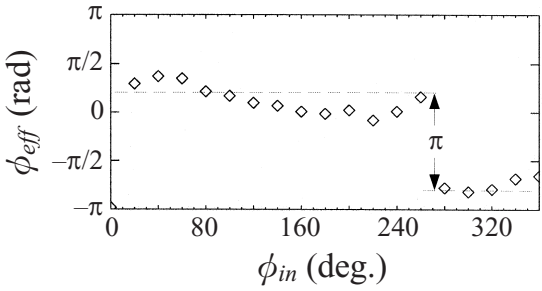

FIGURE 5. (a) Local subharmonic amplitude variation and $(b)$ local effective phase as a function of $\phi_{\text {in }}$ for $u_{f}^{\prime}=0.01 \%, u_{s}^{\prime}=0.1 \%$.

not presented) that the local $\phi_{\text {eff }}$ value strongly depends on the cross-stream location in the shear layer. For this reason, care must be exercised when comparing effective phase results from different experiments. It should also be remembered that the initial effective phase, $\phi_{i n}$, is actually a value imposed at the exit plane of the jet nozzle. Due to the strong lateral spatial gradients in $\phi_{\text {eff }}$, the corresponding initial value in the shear layer will vary accordingly.

In order to demonstrate the local response of subharmonic amplitude to initial F-S phase angle, $\phi_{i n}$, figure $5(a)$ presents measurements of subharmonic amplitude (normalized by the local subharmonic maximum) as obtained at selected $x / \theta_{0}$ locations both upstream and near subharmonic mode saturation as a function of the initial effective phase angle, $\phi_{i n}$. Figure $5(b)$ presents the corresponding local values of effective F-S phase angle, $\phi_{\text {eff }}$, as a function of $\phi_{i n}$. The data of figures $5(a)$ and $5(b)$ correspond to fundamental and subharmonic forcing amplitudes of $u_{f}^{\prime}=0.01 \%$ and $u_{s}^{\prime}=0.1 \%$.

Figure 5(a) shows that at $x / \theta_{0}=50$ the subharmonic amplitude exhibits a clear dependence on the initial effective phase angle, with a maximum near $\phi_{\text {in }}=30^{\circ}$ and a minimum for $\phi_{\text {in }}=220^{\circ}$. Similar subharmonic amplitude behaviour can be observed at the $x / \theta_{0}=60$ station with the local subharmonic amplitude maximum at $\phi_{\text {in }} \approx 40^{\circ}$ and minimum amplitude shifted to $\phi_{\text {in }} \approx 260^{\circ}$. By $x / \theta_{0}=80$ (which is near subharmonic amplitude saturation) there exists a broad range of initial effective phase angles for which the subharmonic amplitude remains virtually constant and very near the maximum value. These values of $\phi_{i n}$ are associated with vortex pairing. Only for a limited range of $\phi_{i n}$ does the subharmonic amplitude decrease. There is a 
cusp centred near $\phi_{i n}=280^{\circ}$ in which the subharmonic amplitude drops drastically. The cusp of reduced subharmonic amplitude (which is associated with vortex tearing) is quite localized in $\phi_{i n}$. This result demonstrates that in the nonlinear region of the flow, vortex pairing is highly favoured (i.e. most $\phi_{i n}$ values will favour vortex pairing). The behaviour shown for $x / \theta_{0}=80$ is very reminiscent of that shown in figure 3(c) of Husain \& Hussain (1995). They observed a cusp at $\phi_{i n}=216^{\circ}$ for higher forcing levels (both fundamental and subharmonic were excited at $0.1 \%$ ). Their experiments at a forcing level of $1 \%$ showed similar behaviour with the cusp occurring at $306^{\circ}$.

Figure 5(b) shows the average local effective phase angle $\phi_{\text {eff }}$ as a function of $\phi_{\text {in }}$ for the same streamwise stations as those presented in figure $5(a)$. At the $x / \theta_{0}=50$ location the local effective phase varies linearly with $\phi_{i n}$. At $x / \theta_{0}=60$ the variation of $\phi_{\text {eff }}$ with $\phi_{\text {in }}$ is observed to be quite nonlinear for $0^{\circ}<\phi_{\text {in }}<120^{\circ}$ and $240^{\circ}<\phi_{\text {in }}<$ $360^{\circ}$. Finally, near $x / \theta_{0}=80$ the effective phase exhibits a strong nonlinear variation with $\phi_{i n}$. In particular, the local effective phase only weakly follows the $\phi_{\text {in }}$ linear variation and appears to lock onto either one of two values. There are two localized $\pi$-phase shifts between these phase plateaux for $\phi_{\text {in }} \approx 0^{\circ}$ and $\phi_{\text {in }} \approx 270^{\circ}$. The latter value is centred near the cusp corresponding to minimum subharmonic amplitude shown in figure 5(a). Figure 5(b) shows that once $\mathrm{F}-\mathrm{S}$ locking occurs, there are two allowed effective F-S phase angles. These are shifted by $\pi$ radians with respect to each other and the values are observed to be largely independent of $\phi_{\text {in }}$ (though they are functions of $x / \theta_{0}$ ).

For the bimodal shear layer excitation condition corresponding to figure $5(a, b)$, two values of $\phi_{i n}$ were selected in order to investigate the streamwise subharmonic evolution. The selected values were $\phi_{\text {in }}=0^{\circ}$ which corresponds to strong subharmonic growth (i.e. vortex pairing) and $\phi_{i n}=260^{\circ}$ which corresponds to subharmonic suppression (i.e. vortex tearing). The streamwise evolution of modal amplitudes of both the fundamental instability, $f_{e}$, and subharmonic, $f_{e} / 2$ for both $\phi_{i n}$ cases are shown in figure 6 (as determined from conventional Fourier analysis). The profound effect that the initial $\mathrm{F}-\mathrm{S}$ phase angle $\phi_{\text {in }}$ can have upon the development of the jet shear layer is apparent from this figure. In the $\phi_{i n}=0^{\circ}$ case, the subharmonic exhibits strong growth and saturates near $x / \theta_{0}=80$. In the $\phi_{i n}=260^{\circ}$ case the subharmonic amplitude is strongly suppressed over the range $60<x / \theta_{0}<120$ and is actually in decay over $55<x / \theta_{0}<80$. The back effect of the subharmonic on the fundamental is also apparent, an aspect not treated in the theory of Monkewitz (1988). Figure 6 also presents the streamwise variation in average effective phase $\phi_{\text {eff }}$ (also determined by Fourier analysis) which behaves quite differently in each case. In both cases, however, the local effective phase angle $\phi_{\text {eff }}$ is observed to vary continuously with $x$. This indicates that the values of the effective phase $\phi_{\text {eff }}$ plateaux shown in figure $5(b)$ for $x / \theta_{0} \approx 80$ are local and will, in general, vary in the streamwise direction. Note, however, that in the region of subharmonic decay $\left(55<x / \theta_{0}<80\right)$ in figure 6 , the effective phase curves for the two cases are separated by $\pi$ radians. The theoretical model, to be presented in $\S 5$, will explain the existence of the two possible values for the local effective phase and the $\pi$-dislocation in phase separating the two. Figure 6 indicates that while the particular value of $\phi_{\text {eff }}$ associated with the plateaux in phase vary in the streamwise direction, the local values associated with pairing and tearing remain separated by $\pi$ radians in the region of strongest subharmonic suppression.

In the bimodal forcing experiments the Morlet wavelet transform was applied to the experimentally obtained streamwise velocity fluctuation time histories. The phase time histories $\phi_{f}(t)$ and $\phi_{s}(t)$ were extracted by application of equation (2.1) at the appropriate fixed $\kappa$. The associated effective phase time history was then obtained 

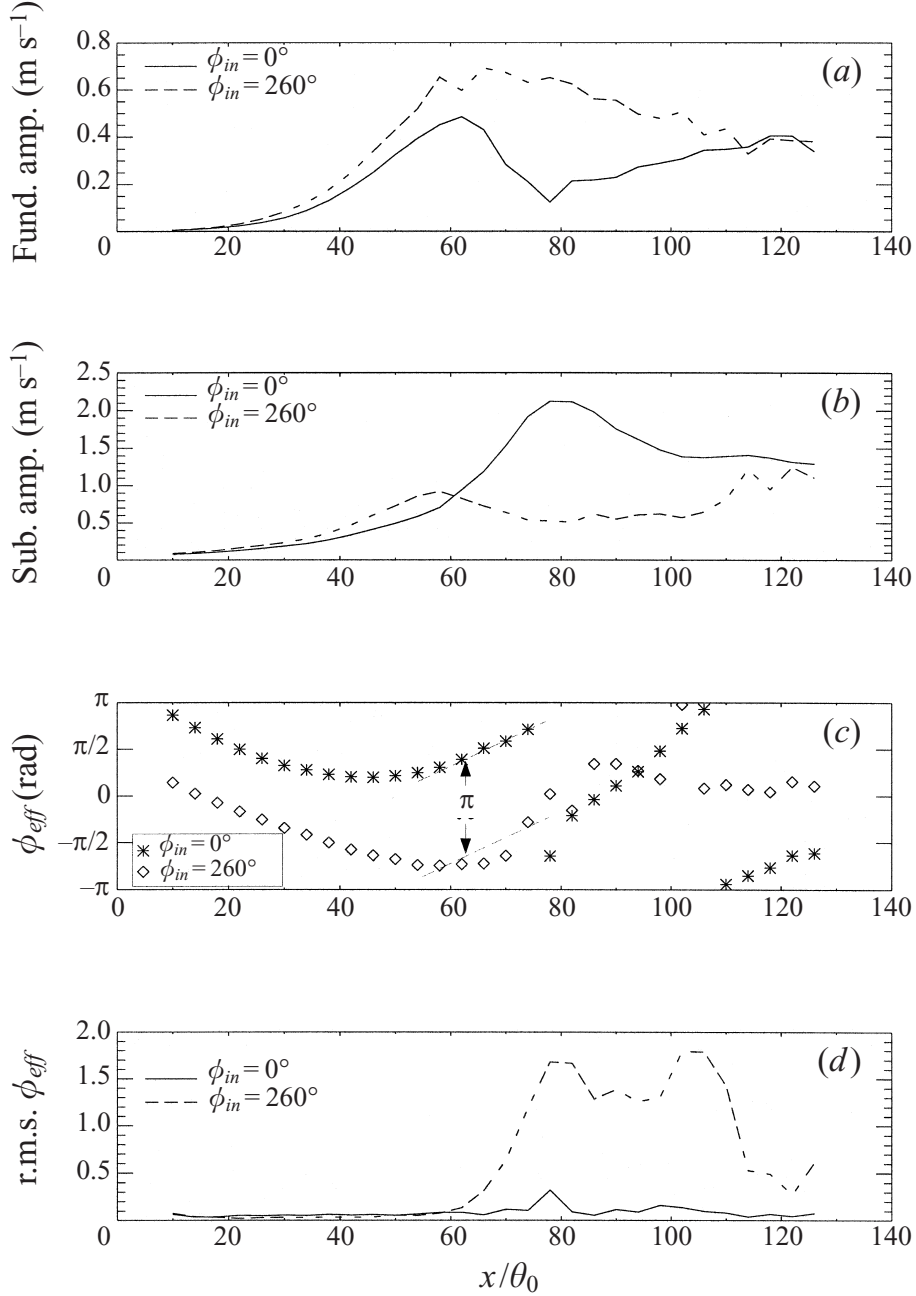

FIGURE 6. Streamwise evolution of fundamental amplitude, subharmonic amplitude, $\phi_{\text {eff }}$ and r.m.s. of $\phi_{\text {eff }}$ for $\phi_{\text {in }}=0^{\circ}$ (pairing) and $\phi_{\text {in }}=260^{\circ}$ (tearing), $u_{f}^{\prime}=0.01 \%, u_{s}^{\prime}=0.1 \%$.

as $\phi_{\text {eff }}(t)=\phi_{f}(t)-2 \phi_{s}(t)$. The root-mean-square (r.m.s.) value of the $\phi_{\text {eff }}(t)$ time history provides an indication of regions where temporal phase variations are most significant. The lowest plot of figure 6 presents the resulting streamwise variation of the r.m.s. of the effective phase as determined from the wavelet analysis. This provides a measure of the degree of temporal variation of the measured effective $\mathrm{F}-\mathrm{S}$ phase $\phi_{\text {eff }}$. As shown in the example presented in figure 1, the finite time resolution of the wavelet transform will have the tendency to smooth a sudden shift in phase. In effect, the step change in phase is low-pass filtered. As such, one could argue regarding the physical relevance of the r.m.s. value of the effective phase. However, there is no question that it represents a good indicator of those $x$ locations where $\phi_{\text {eff }}$ exhibits temporal variation and this is our only purpose in presenting the r.m.s. phase values shown in figure 6 . The streamwise variation in r.m.s. is presented for both excitation cases. In the case $\phi_{i n}=0^{\circ}$, which favours strong subharmonic growth (i.e. vortex pairing) the r.m.s. $\approx 0$ for all $x$ indicating the inherent temporal stability 

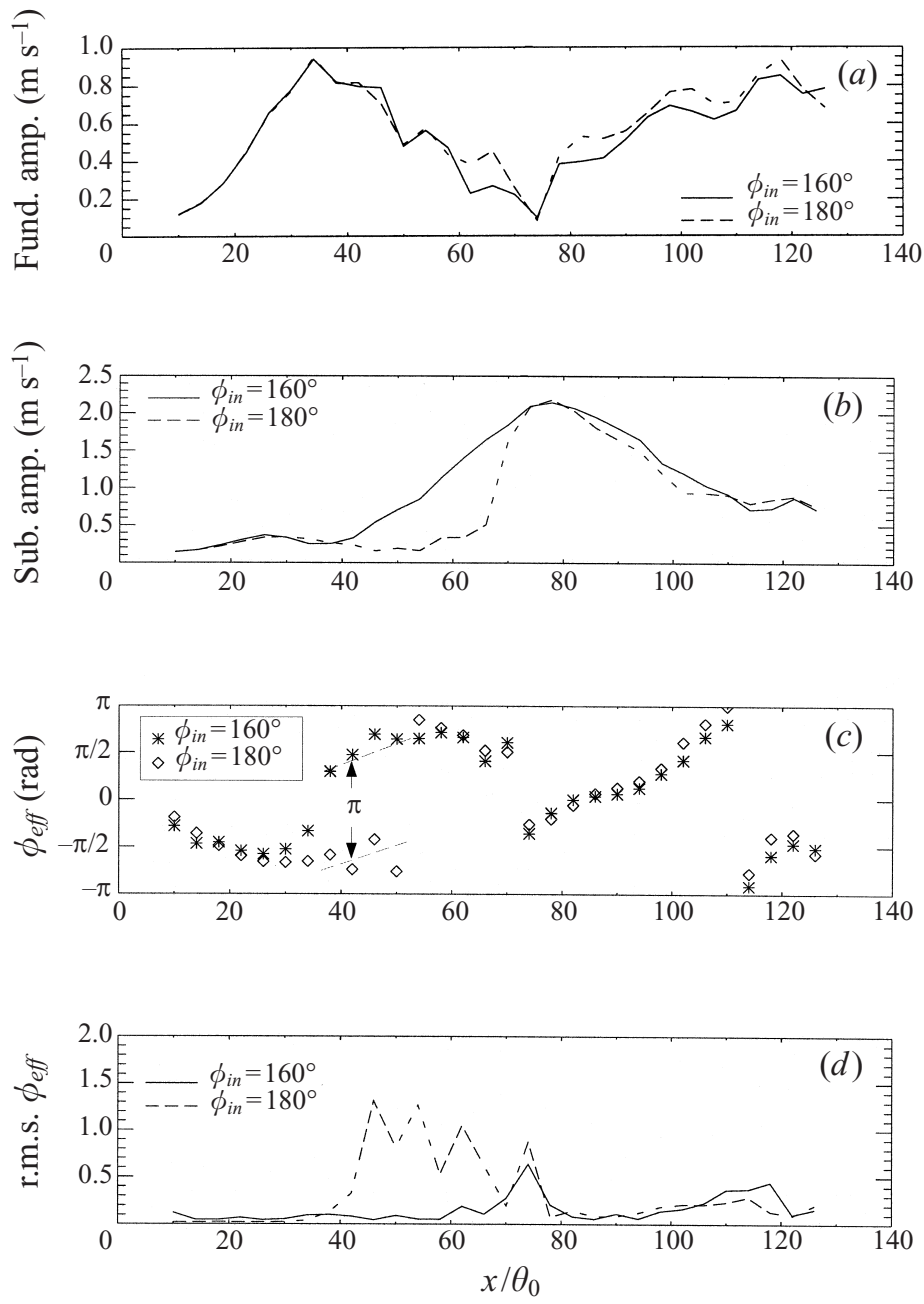

FIGURE 7. Streamwise evolution of fundamental amplitude, subharmonic amplitude, $\phi_{\text {eff }}$ and r.m.s. of $\phi_{\text {eff }}$ for $\phi_{\text {in }}=160^{\circ}$ (pairing) and $\phi_{\text {in }}=180^{\circ}$ (tearing), $u_{f}^{\prime}=u_{s}^{\prime}=0.1 \%$.

of the local effective F-S phase. In the second case, $\phi_{i n}=260^{\circ}$, which corresponds to subharmonic suppression (i.e. vortex tearing), the r.m.s. is observed to be significantly increased for $60<x / \theta_{0}<120$ which is the same streamwise region which exhibits subharmonic suppression. This implies that this region is also characterized by a high level of temporal variation in phase. We will consider this region further when we present additional wavelet analysis results in the next section.

Figure 7 presents the streamwise amplitude variation of the fundamental and subharmonic modes, the local effective F-S phase (as determined by Fourier analysis) and the r.m.s. of the effective phase (obtained via wavelet analysis as described above) for bimodal excitation at amplitudes $u_{f}^{\prime}=u_{s}^{\prime}=0.1 \%$. Two cases are shown: $\phi_{i n}=160^{\circ}$ and $\phi_{i n}=180^{\circ}$. Due to the higher fundamental excitation amplitude, the fundamental saturates at $x / \theta_{0} \approx 35$ in both cases which is well upstream of the saturation location shown in figure 6. Again, significant disparities in the streamwise evolution of the subharmonic instability may be observed. In particular, the subharmonic is suppressed in the region $40<x / \theta_{0}<70$ for the $\phi_{\text {in }}=180^{\circ}$ case. In contrast, the $\phi_{\text {in }}=160^{\circ}$ 


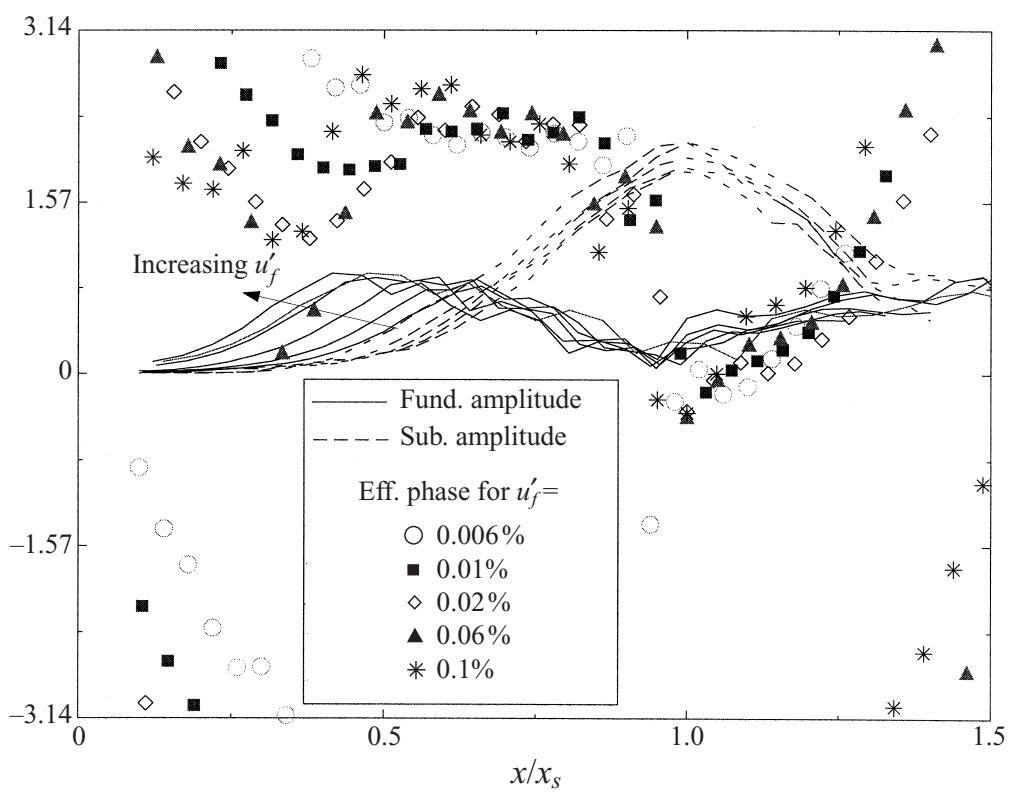

FIGURE 8. Streamwise evolution of fundamental amplitude, natural subharmonic amplitude and $\phi_{\text {eff }}$ for several $u_{f}^{\prime} ; u_{s}^{\prime}=0$ in each case.

case is characterized by strong subharmonic growth. As in figure 6 , there is a $\pi$ difference in local $\phi_{\text {eff }}$ values between the two cases in the region $40<x / \theta_{0}<50$ which is associated with strong subharmonic suppression. Note, also that for the $\phi_{\text {in }}=180^{\circ}$ case, the r.m.s. exhibits elevated values in this region as was the case in figure 6 . In contrast, the $\phi_{i n}=160^{\circ}$ case shows values of r.m.s. $\approx 0$ indicating little temporal variation in effective phase. This leads to the conclusion that the subharmonic suppression is characterized by an inherently unsteady effective phase behaviour. Comparison of figures 6 and 7 also indicates that the $\phi_{\text {in }}$ giving rise to subharmonic suppression is clearly dependent upon the initial excitation level.

\subsection{Single mode forcing experiments: natural subharmonic evolution}

In the second series of experiments, only the fundamental instability wave was artificially excited. Since there is no artificial forcing at the subharmonic frequency, the subharmonic mode will develop naturally and eventually reach an optimum phase relationship with the fundamental mode. These experiments focus on the natural aspects of the $\mathrm{F}-\mathrm{S}$ interaction which will be shown to be inherently unsteady. Both standard Fourier and the Morlet wavelet transformations were applied in order to process the hot-wire signals.

In figure 8 the streamwise evolution of both fundamental and subharmonic modes as well as the local average effective phase angle $\phi_{\text {eff }}$ are presented for several different fundamental excitation amplitudes spanning two orders of magnitude: $0.006 \%<u_{f}^{\prime}<$ $0.1 \%$. The subharmonic is not artificially excited; $u_{s}^{\prime}=0$. There will, of course, be a naturally occurring subharmonic perturbation near the nozzle lip due to upstream feedback. The streamwise coordinate $x$ is non-dimensionalized by $x_{s}$ which is the location of subharmonic mode saturation for each case. All measurements were taken along the line of the local subharmonic cross-stream maxima. Standard Fourier 
analysis techniques were utilized to obtain the modal amplitudes and average local effective phase shown in figure 8 .

In the initial region $x / x_{s}<0.5$, a wide range of $\phi_{\text {eff }}$ values is observed and these clearly depend on fundamental excitation level. The fundamental wave amplitude saturates farther upstream with increased initial excitation amplitude, $u_{f}^{\prime}$, as indicated. However, for $x / x_{s}>0.5$, the local effective phase angles are found to converge and all exhibit a similar streamwise variation. In this region the fundamental mode decays, the subharmonic mode is strongly amplified and all $\phi_{\text {eff }}$ values remain near a value of $3 \pi / 4 \approx 2.4$. Note that this is quite near the local values of $\phi_{\text {eff }}$ which were found to be associated with strong subharmonic amplification in the bimodal forcing experiments (figures 6 and 7) In fact, there is a strong similarity in the streamwise variation in $\phi_{\text {eff }}$ observed in figures 8,7 and 6 . Figure 8 shows that just upstream of subharmonic mode saturation the fundamental reaches minimum amplitude and $\phi_{\text {eff }}$ exhibits a sudden drop to a value near zero.

The data of figure 8 were used to determine whether a critical fundamental amplitude exists for onset of $\mathrm{F}-\mathrm{S}$ resonance. The criterion used to characterize initiation of resonance was the departure of the local effective phase $\phi_{\text {eff }}$ from its initial streamwise variation and its approach toward the converged effective phase values shown in figure 8 . With the exception of the highest excitation case, the fundamental amplitudes corresponding to these streamwise locations were found to be at a value of $0.016 U_{0}$ within experimental uncertainty. This corresponds well to the value of $0.015 U_{0}$ predicted by the analysis of Monkewitz (1988) for a mixing layer with velocity ratio $R=1$.

These results show that for a wide range of initial fundamental excitation amplitudes the shear layer will, after the fundamental reaches sufficient amplitude (approximately $\left.0.016 U_{0}\right)$, begin to exhibit a similar nonlinear variation of $\phi_{\text {eff }}$ as a function of $x / x_{s}$. In a sense, with sufficient downstream distance the effect of initial conditions is gradually lost and a common phase and subharmonic amplitude behaviour is observed. Sustained vortex tearing must be artificially forced via bimodal excitation and is not observed in the results depicted in figure 8 . From figure 8 one can also conclude that $\phi_{\text {eff }}$ at $x=0$ is a function of initial fundamental excitation amplitude and therefore there is no unique optimum $\phi_{\text {in }}$ value for the jet shear layer. An amplitude dependence of $\phi_{i n}$ for subharmonic suppression was also noted in the bimodal forcing experiments.

Two cases of low-level fundamental excitation were selected in order to investigate the temporal aspects of the F-S interaction. The streamwise variation of both the $f_{e}$ and $f_{e} / 2$ modal amplitudes as well as the local average effective phase $\phi_{e f f}$ and its r.m.s. are presented in figure 9 for the case of extremely low-level fundamental excitation, $u_{f}^{\prime}=0.002 \%$ (i.e. slightly above natural background disturbances within a narrow frequency band centred on $f_{e}$ ). It is apparent from the high values of r.m.s. that the effective phase exhibits a high degree of temporal unsteadiness, especially in the region extending to $x / \theta_{0} \approx 80$. In the region $x / \theta_{0}>80$ the temporal $\phi_{\text {eff }}$-fluctuations are significantly reduced. This indicates that the fundamental and natural subharmonic mode establish a certain degree of resonant phase locking in this region. This is confirmed by the streamwise variation in the cross-bicoherence which is presented in figure 9. Note the large increase in cross-bicoherence $b^{2}\left(f_{e},-f_{e} / 2\right)$ commencing near $x / \theta_{0} \approx 80$ which confirms the enhanced phase locking between the fundamental and subharmonic waves at this location. However, even in the region of strongest subharmonic growth, there is still an inherent unsteadiness and the r.m.s. remains greater than zero. This is also shown in the streamwise amplitude variation of the 

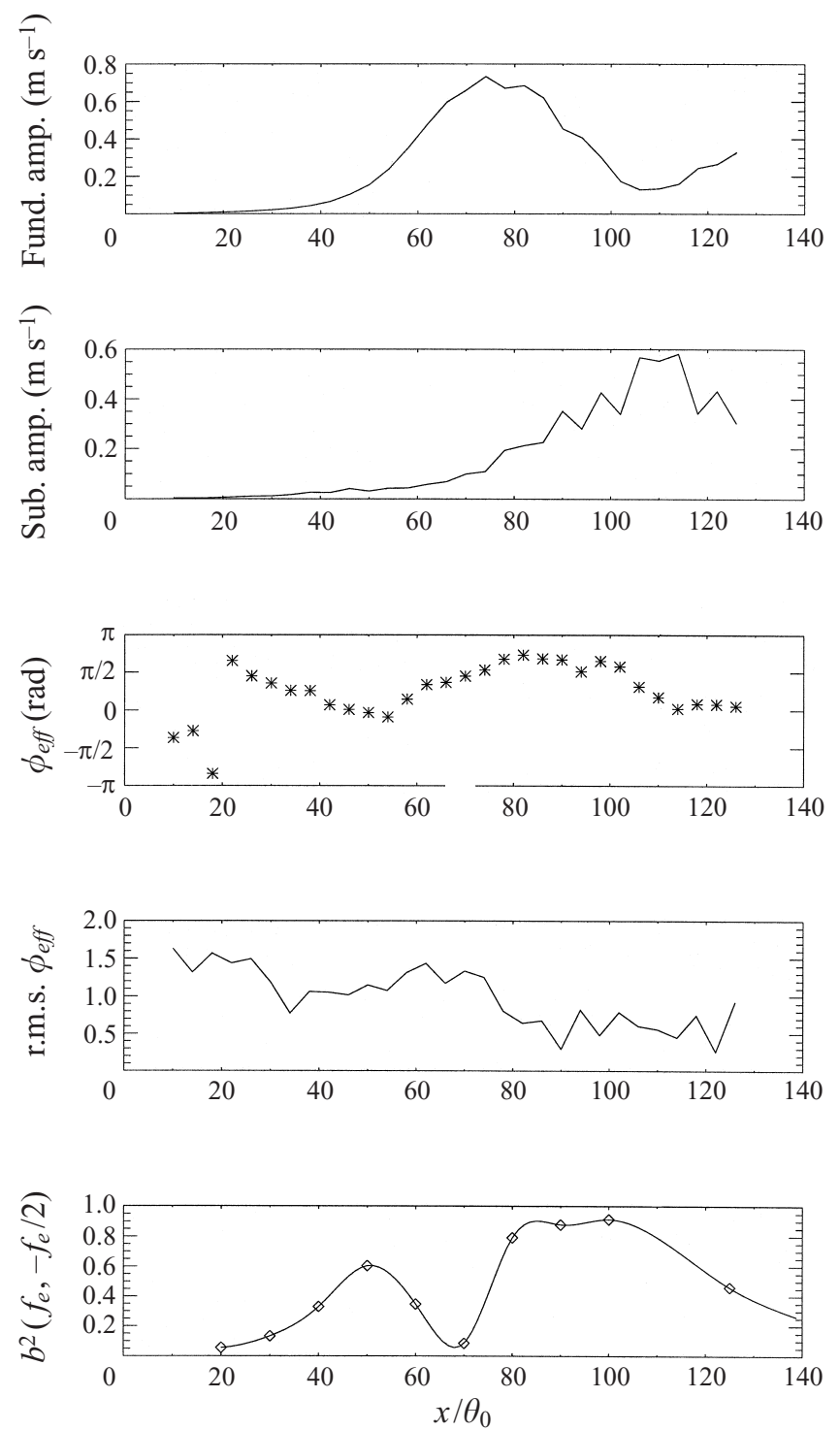

FIGURE 9. Streamwise variation of fundamental and subharmonic amplitudes, $\phi_{\text {eff }}$, r.m.s. of $\phi_{e f f}$ and cross-bicoherence for $u_{f}^{\prime}=0.002 \%, u_{s}^{\prime}=0$.

subharmonic which appears quite jagged. The amplitude was computed by standard Fourier analysis with an ensemble average over twenty five data blocks. Due to the inherent temporal variation of the natural subharmonic this was insufficient in this case to achieve a smooth spectral estimate. This behaviour may be contrasted with the case of moderate fundamental excitation $u_{f}^{\prime}=0.01 \%, u_{s}^{\prime}=0$ which is presented in figure 10. In this case the subharmonic amplitude reaches a much higher saturation value and the spectral estimates are smooth. The corresponding r.m.s. values are near zero which indicates a smaller degree of the temporal phase variation. This indicates that the $\mathrm{F}-\mathrm{S}$ phase locking is much stronger in this case and the increased amplification of the subharmonic results. 

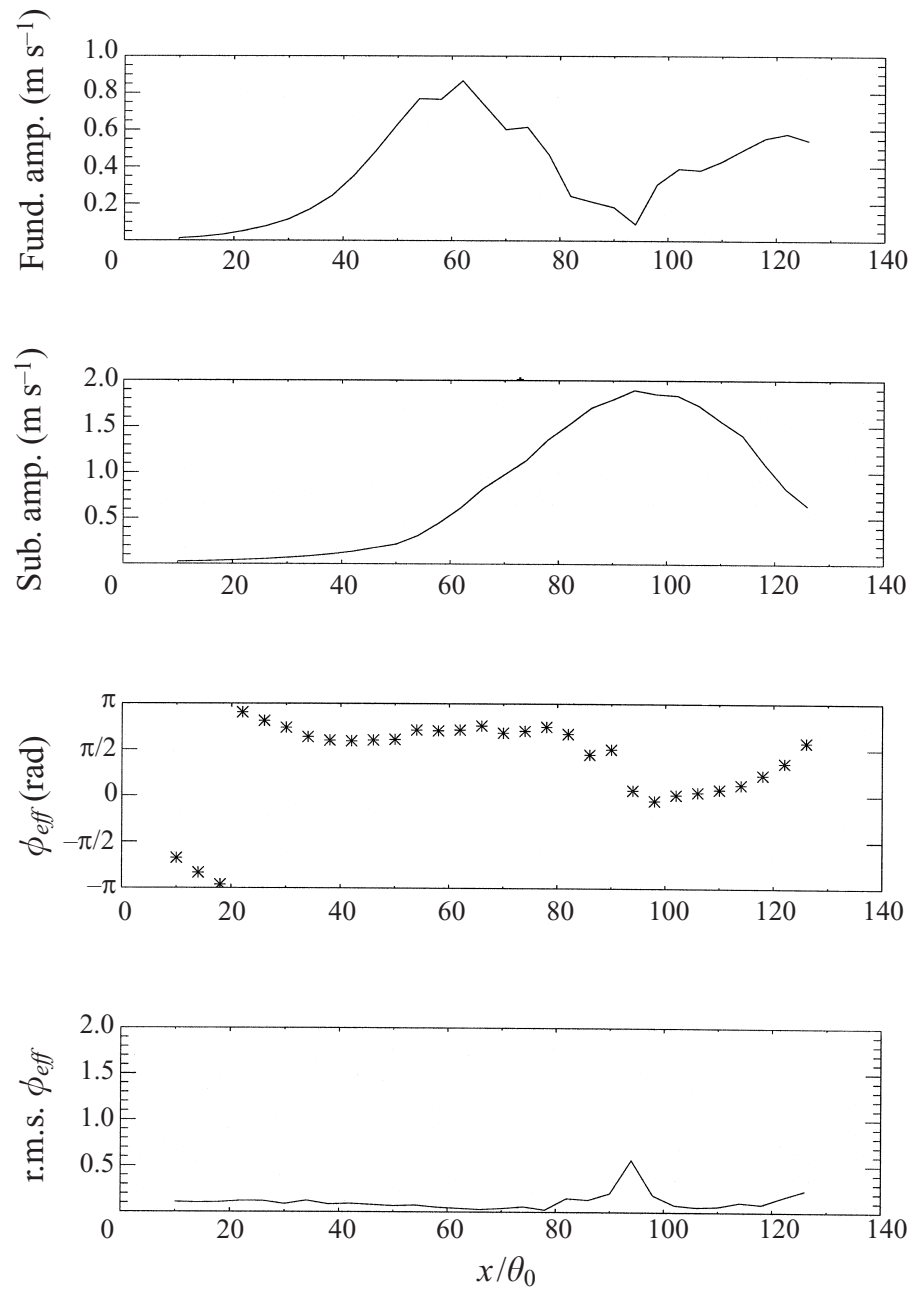

FIGURE 10. Streamwise variation of fundamental and subharmonic amplitudes, $\phi_{\text {eff }}$ and r.m.s. of $\phi_{\text {eff }}$ for $u_{f}^{\prime}=0.01 \%, u_{s}^{\prime}=0$.

The results presented to this point demonstrate that temporal variation in effective phase behaviour (as evidenced by high r.m.s. values of effective phase) may be expected in bimodal forcing experiments in those regions characterized by subharmonic mode suppression even if the forcing amplitudes are quite large. In addition, temporal phase variation is the rule in experiments involving low-amplitude forcing of the fundamental and natural development of the subharmonic. In order to properly investigate these temporal aspects of the shear layer dynamics, a wavelet transformation technique was applied in the analysis of the hot-wire signals. To demonstrate the effectiveness of the wavelet transform for this purpose, consider the modulus of the Morlet wavelet transform of the shear layer hot-wire signal which is presented in figure 11. The signal for figure 11 corresponds to $u_{f}^{\prime}=0.002 \%, u_{s}^{\prime}=0$ (no artificial subharmonic excitation) at $x / \theta_{0}=90$ (i.e. just upstream of subharmonic saturation, see figure 9). The abscissa is time $\tau(\mathrm{s})$ and the ordinate is $f(\kappa)=b \kappa / 2 \pi$ (and is expressed in $\mathrm{Hz}$ ). The use of frequency units on the ordinate is potentially misleading and it must be emphasized that this should not be interpreted as implying periodicity. 


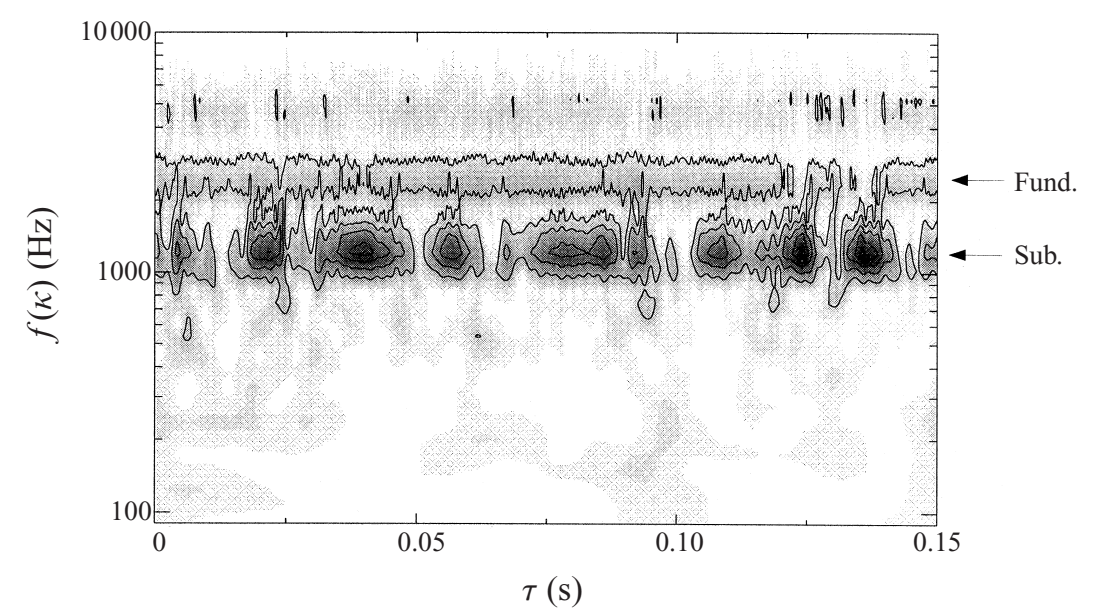

FIGURE 11. Morlet wavelet transform modulus $\left|G_{\Psi}(\kappa, \tau)\right|$ at $x / \theta_{0}=90, u_{f}^{\prime}=0.002 \%, u_{s}^{\prime}=0$.

Rather this is a convenient way to relate an event from the wavelet analysis to the standard Fourier analysis results. The modulus of the signal is plotted in the form of a shaded contour plot with darker shading associated with higher levels of the signal modulus. The $f(\kappa)$ values associated with the fundamental and subharmonic instabilities are identified on the ordinate. The temporal variation of the subharmonic modulus is especially apparent. The modulus of the fundamental is, by comparison, fairly constant. Maxima and minima of the subharmonic modulus do not appear correlated with any similar variation in the fundamental. Figure 11 clearly demonstrates the inherent temporal variation that characterizes the natural subharmonic instability.

As in the example presented in figure 1 , at any selected $x$-station one can obtain a time series corresponding to the amplitude envelope and phase of individual shear layer modes by taking a 'slice' of the wavelet transform (e.g. like figure 11) at the appropriate $f(\kappa)$. Since the focus of this paper is on the fundamental and subharmonic instabilities, this manner of presenting the wavelet transformation results is more appropriate than full contour plots in $f(\kappa), \tau$ space. Consequently, in the remainder of the paper wavelet results will be presented in terms of derived amplitude and phase time series for the fundamental and subharmonic instability waves. Recall also that it is the phase term $\phi$ and not $\theta$ that is of primary interest.

Figure 2 presented the time variation of the amplitude and phase of the subharmonic instability at $x / \theta_{0}=110$ for the case of $u_{f}^{\prime}=0.002 \%$ and $u_{s}^{\prime}=0$. Figure 9 shows this location to be in a region of natural subharmonic growth although the r.m.s. of the effective phase $>0$. Examination of figure 2 reveals an interesting temporal behaviour of the subharmonic mode which was already highlighted at the beginning of this section. It shows the existence of two steady values of subharmonic phase with intermittent $\pi$-jumps between them. These phase jumps are observed to be well correlated with local drops in the subharmonic amplitude. In contrast, time intervals of stable subharmonic phase are associated with elevated subharmonic amplitude. Although not presented in figure 2, the fundamental amplitude and phase do not show any behaviour of this sort; both exhibit only very small temporal variation. The $\pi$-jumps in subharmonic phase give rise to corresponding intermittent disruptions in the local effective phase angle, $\phi_{\text {eff }}$, which is also presented in figure 2 . The resonant 

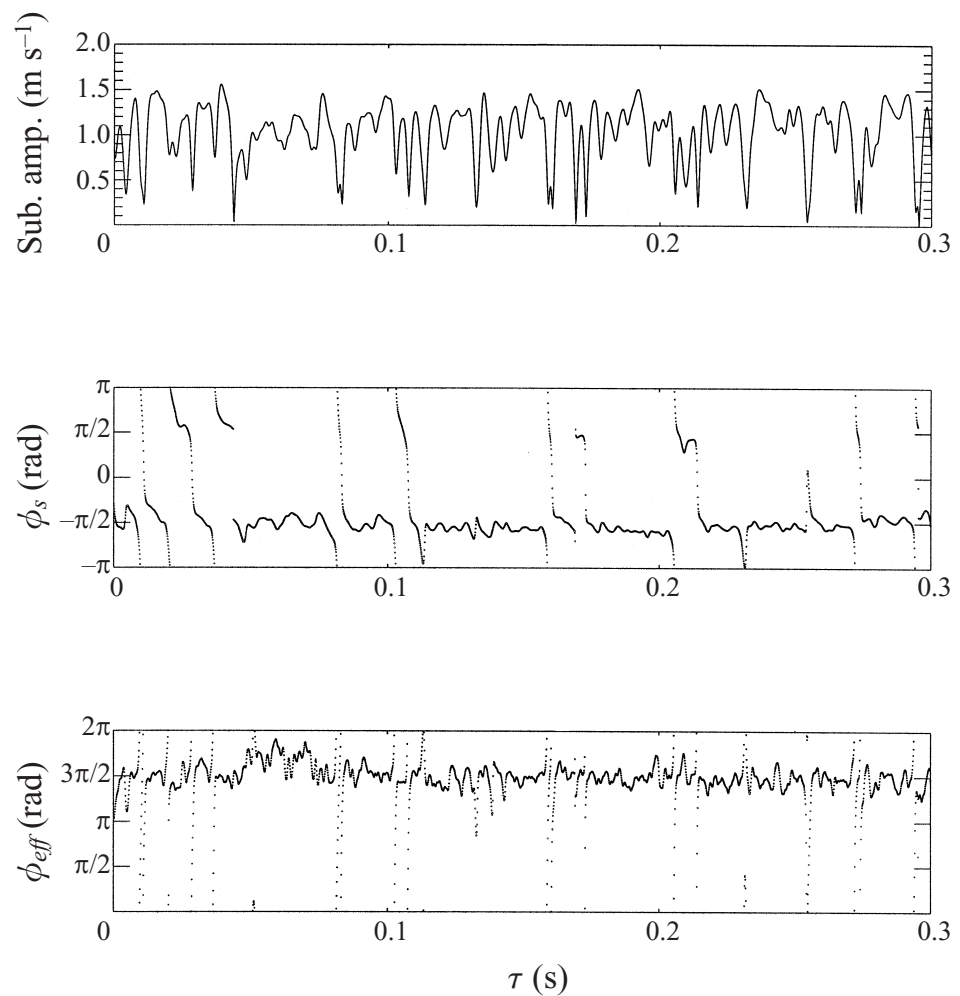

FIGURE 12. Temporal variation of subharmonic amplitude, phase and effective phase: $u_{f}^{\prime}=0.01 \%$, $u_{s}^{\prime}=0, x / \theta_{0}=70$.

phase locking between fundamental and subharmonic is shown to be intermittently disrupted due to the unsteady phase behaviour of the subharmonic.

Figure 12 shows wavelet transform results obtained at $x / \theta_{0}=70$ for a higher fundamental excitation amplitude, $u_{f}^{\prime}=0.01 \%$ and $u_{s}^{\prime}=0$. As in the previous case, the subharmonic phase is characterized by stable values which are interrupted by $\pi$-shifts that correlate with minima in the subharmonic amplitude. The fundamental is, by comparison, steady in amplitude and phase. However, a notable difference between the behaviour shown in figures 2 and 12 is that for the higher-amplitude forcing case, the subharmonic phase exhibits a clear preference for one of the phase plateaux with little time spent at the other: $92 \%$ residence time at one phase value and only $8 \%$ at the other. In figure 2, both plateaux are nearly equally probable: $52 \%$ at one phase value and $48 \%$ at the other. This amplitude-dependent effect will be explained by the dynamical systems model to be presented in $\S 5$. The phase dislocation behaviour shown in figures 2 and 12 was found to be typical of the regions of the flow corresponding to $80<x / \theta_{0}<150$ for both low- and moderate-level fundamental mode excitation, which corresponds to the region of the strongest $\mathrm{F}-\mathrm{S}$ interaction.

Recall that in the experiments involving moderate- and even high-level bimodal forcing, regions of subharmonic mode suppression were found to be associated with unsteady effective phase behaviour as evidenced by high values of the r.m.s. of the effective phase. Application of the Morlet wavelet transformation to those regions shows a similar unsteady phase behaviour to that described above. For example, 

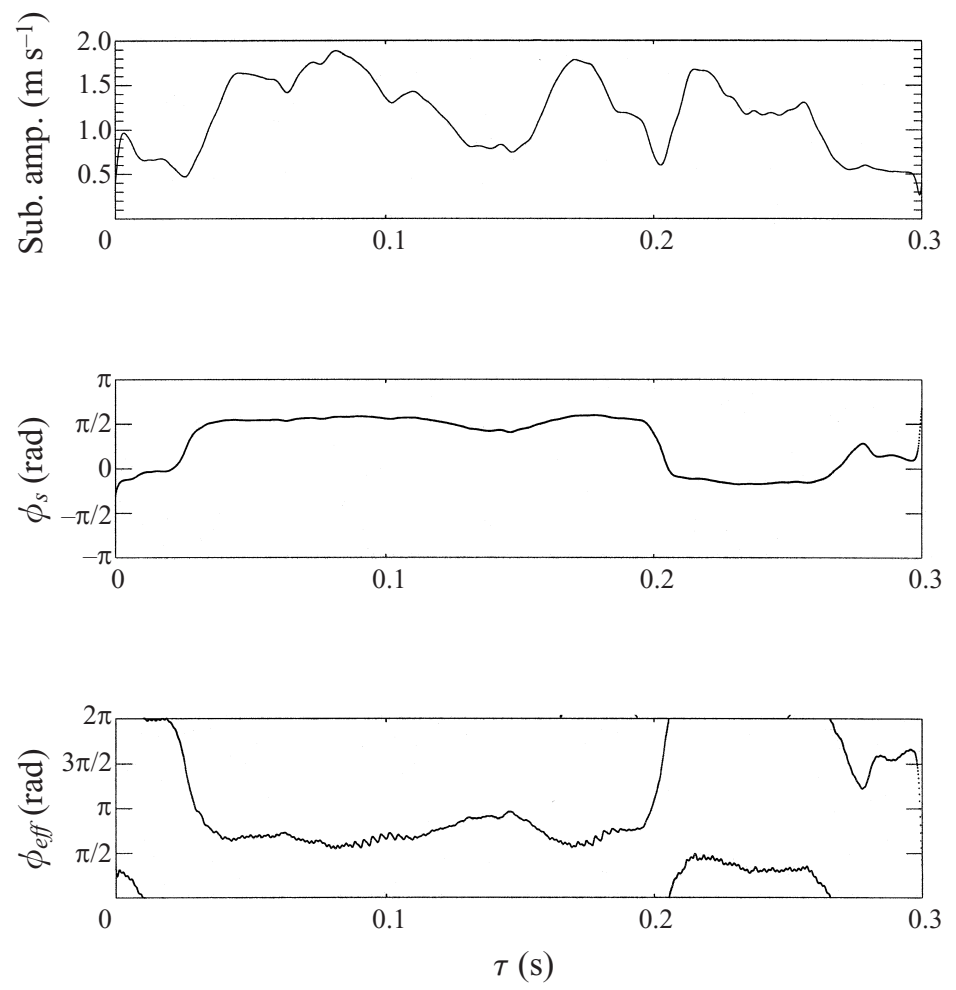

FIGURE 13. Temporal variation of subharmonic amplitude, phase and effective phase: $u_{f}^{\prime}=0.01 \%$, $u_{s}^{\prime}=0.1 \%, x / \theta_{0}=80, \phi_{\text {in }}=260^{\circ}$ (tearing).

figure 13 shows Morlet wavelet transformation results corresponding to bimodal excitation with $u_{f}^{\prime}=0.01 \%, u_{s}^{\prime}=0.1 \%$ and $\phi_{i n}=260^{\circ}$ at location $x / \theta_{0}=80$. This case corresponds to the strong subharmonic suppression shown in figure 6. Figure 13 indicates that while the amplitude envelope and phase of the fundamental are quite steady, the subharmonic instability has both temporal amplitude and phase behaviour which is similar in character to that shown in figure 2 for the natural subharmonic. In this case the temporal behaviour is characterized by longer periods of stable subharmonic phase at two possible values which are interrupted by sudden, intermittent phase shifts. These correlate well with local minima in subharmonic amplitude. Comparison of figures 6 and 13 suggests that these temporal phase events are associated with the vortex tearing process.

\section{Theoretical model}

\subsection{Model formulation}

In an attempt to model the temporal behaviour observed in the experiments, one can take two approaches. One can utilize a wave formulation and apply nonlinear stability analysis, which, by necessity will be restricted to weak nonlinearity. An alternative approach is to recognize that an essential aspect of the flow in the region in question (say, $70<x / \theta_{0}<150$ ) is the mutual interaction of spanwise shear layer vortices. Advantages of a vortex dynamics approach are that a fully nonlinear model can be developed and the results are easy to interpret within the context of large-scale 


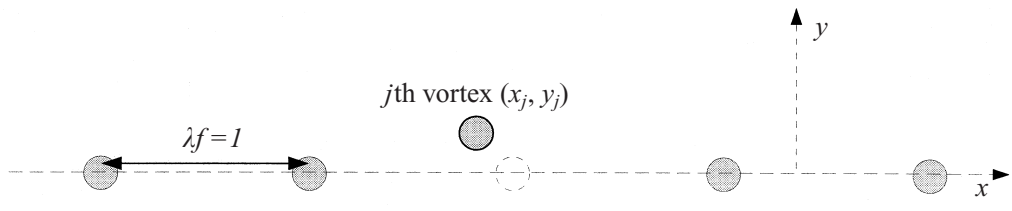

FIGURE 14. Schematic of the array of vortices.

structures. In this section a nonlinear model, based on the theory of interacting vortices as outlined in classic texts by Lamb (1945) and Batchelor (1967) is developed in order to explain the temporal behaviour observed in the experiments. The approach we take is similar to that utilized by Betyayev, Gaifullin \& Gordeyev (1994) to build a fully nonlinear model simulating the evolution of a spiral vortex sheet.

As an idealization, the shear layer near the roll-up location is replaced by an infinite array of two-dimensional vortices, each having a solid-like core and the same circulation $\Gamma$. In a coordinate system convecting with the vortices $x-U_{c} t \rightarrow x$, where $U_{c}$ is the mean convective speed of the vortices. The geometry and the coordinate system are shown in figure 14. Using Taylor's hypothesis, in this moving frame of reference the spatial coordinate $x$ in the model actually corresponds to the time coordinate in the physical problem. Also the key parameter of the system now becomes the fundamental wavelength $\lambda_{f}$ (the distance between the vortices), instead of the fundamental passage frequency.

The azimuthal velocity induced by a single vortex $u_{\theta}$ as a function of a distance from its centre $r$ is taken as

$$
u_{\theta}=\frac{\Gamma}{2 \pi} \frac{r}{a^{2}+r^{2}},
$$

where $a$ defines the radius of the core. The location of each particular vortex is defined by a coordinate pair $\left(x_{j}, y_{j}\right)$. For the case of a single row of equally spaced vortices with $\lambda_{f}=1\left(x_{j}=j, y_{j}=0\right)$, the $u$-component of the flow field is given by (Lamb 1945)

$$
\begin{aligned}
u_{0}(x, y, a) & =\sum_{j=-\infty}^{+\infty} \frac{\Gamma}{2 \pi} \frac{y}{a^{2}+y^{2}+(x-j)^{2}} \\
& =\frac{\Gamma y}{2 \sqrt{a^{2}+y^{2}}} \frac{\sinh \left(2 \pi \sqrt{a^{2}+y^{2}}\right)}{\cosh \left(2 \pi \sqrt{a^{2}+y^{2}}\right)-\cos (2 \pi x)}
\end{aligned}
$$

with the time mean profile $\bar{U}(y)=\int_{0}^{2 \pi} u(x, y) \mathrm{d} x=\Gamma y /\left(2\left(a^{2}+y^{2}\right)^{1 / 2}\right)$.

The dynamics of the system is governed by the following system of ODEs (Batchelor 1967; Lamb 1945):

$$
\frac{\mathrm{d} x_{j}}{\mathrm{~d} t}=-\frac{\Gamma}{2 \pi} \sum_{k \neq j} \frac{y_{k}-y_{j}}{r_{k j}^{2}}, \quad \frac{\mathrm{d} y_{j}}{\mathrm{~d} t}=\frac{\Gamma}{2 \pi} \sum_{k \neq j} \frac{x_{k}-x_{j}}{r_{k j}^{2}},
$$

where $r_{k j}^{2}=a^{2}+\left(x_{k}-x_{j}\right)^{2}+\left(y_{k}-y_{j}\right)^{2}$. For simplicity, but without loss of generality, we take $\Gamma$ to be $2 \pi$. The above system is the Hamiltonian system

$$
\frac{\mathrm{d} x_{j}}{\mathrm{~d} t}=-\frac{\partial H_{\infty}}{\partial y_{j}}, \quad \frac{\mathrm{d} y_{j}}{\mathrm{~d} t}=\frac{\partial H_{\infty}}{\partial x_{j}},
$$




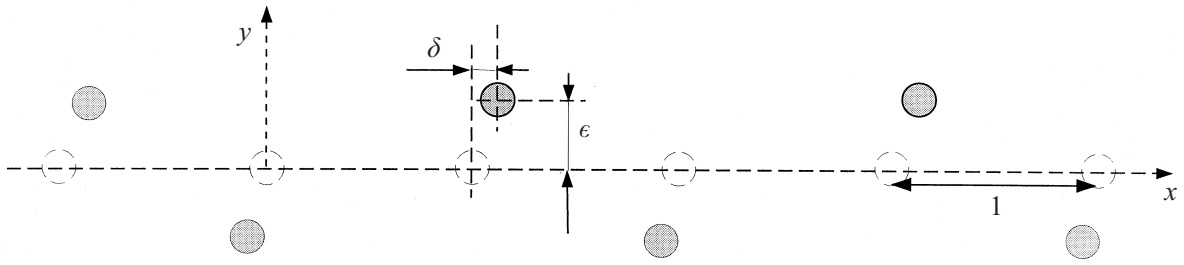

FIGURE 15 . The geometry of the perturbed vortex system.

with

$$
H_{\infty}\left(x_{j}, y_{j}\right)=-\frac{1}{2} \sum_{j} \sum_{k \neq j} \log \left(r_{k j}^{2}\right) .
$$

Consider, in particular, a line of vortices equally spaced along the $x$-axis with unity distance between them $\left(\lambda_{f}=1\right)$ as shown in figure 15. Introduce a subharmonic perturbation with $\lambda_{s}=2 \lambda_{f}=2$ as

$$
x_{j}=j+\delta \cos \left(2 \pi j / \lambda_{s}\right), \quad y_{j}=\varepsilon \cos \left(2 \pi j / \lambda_{s}\right) .
$$

Now the evolution of the system is characterized by the two new variables $(\delta(t), \varepsilon(t))$. Formally the sum (5.5) does not exist for this system, but equals $-\infty$. However, this problem is eliminated by subtracting the undisturbed $H_{\infty}(j, 0)$ from $H_{\infty}$, since we are only interested in the gradient of $H_{\infty}$. After substituting (5.6) into (5.4) and with considerable algebra the system (5.4) can be rewritten for the new variables as

$$
\frac{\mathrm{d} \delta}{\mathrm{d} t}=-\frac{\partial H_{\infty}}{\partial \varepsilon}, \quad \frac{\mathrm{d} \varepsilon}{\mathrm{d} t}=\frac{\partial H_{\infty}}{\partial \delta},
$$

with

$$
H_{\infty}=-\frac{1}{2} \sum_{n=-\infty}^{n=+\infty} \log \left\{\left[1+\frac{4\left(\varepsilon^{2}+\delta^{2}\right)}{a^{2}+j^{2}}\right]^{2}-\left[\frac{4 \delta j}{a^{2}+j^{2}}\right]^{2}\right\}, \quad j=2 n+1 .
$$

Thus $(\delta, \varepsilon)$ is the pair of canonical variables for the system. Carrying out the summation of (5.8) (see the Appendix for details), one can obtain the following analytical expression for the Hamiltonian, $H_{\infty}(\delta, \varepsilon)$ :

$$
H_{\infty}(\delta, \varepsilon)=-\frac{1}{2} \log \left[\cos ^{2}(\pi \delta)+\sinh ^{2}\left(\pi \sqrt{(a / 2)^{2}+\varepsilon^{2}}\right)\right] .
$$

Now the discrete system (5.4) is replaced by the continuous system (5.7) with $H_{\infty}$ in the form of (5.9), which is much more amenable to theoretical analysis. Moreover, due to the symmetry of (5.7), the numerical instability of (5.4) is removed from the system and the physical behaviour of this configuration of vortices can be unambiguously investigated. For Hamiltonian systems, $H_{\infty}$ is the invariant of the system (the energy invariant), e.g. $H_{\infty}(\delta, \varepsilon)=$ const. along trajectories $(\delta(t), \varepsilon(t))$. In other words, the isolines of $H_{\infty}$ show the system behaviour in phase space and this is depicted in figure $16(a)$ and in greater detail in figure $16(b)$.

The system has three physically distinct fixed points: two centre points at $\left( \pm \frac{1}{2}, 0\right)$ and one saddle point at $(0,0)$. The saddle point corresponds to the instability of the vortex line, while the two centres show the existence of two regimes where the system of vortices form interacting pairs. The resulting locations of vortex pairs in both regimes are shifted with respect to each other a distance of $\lambda_{s} / 2=1$.

The velocity field can be found by noticing that the vortex system is a superposition 


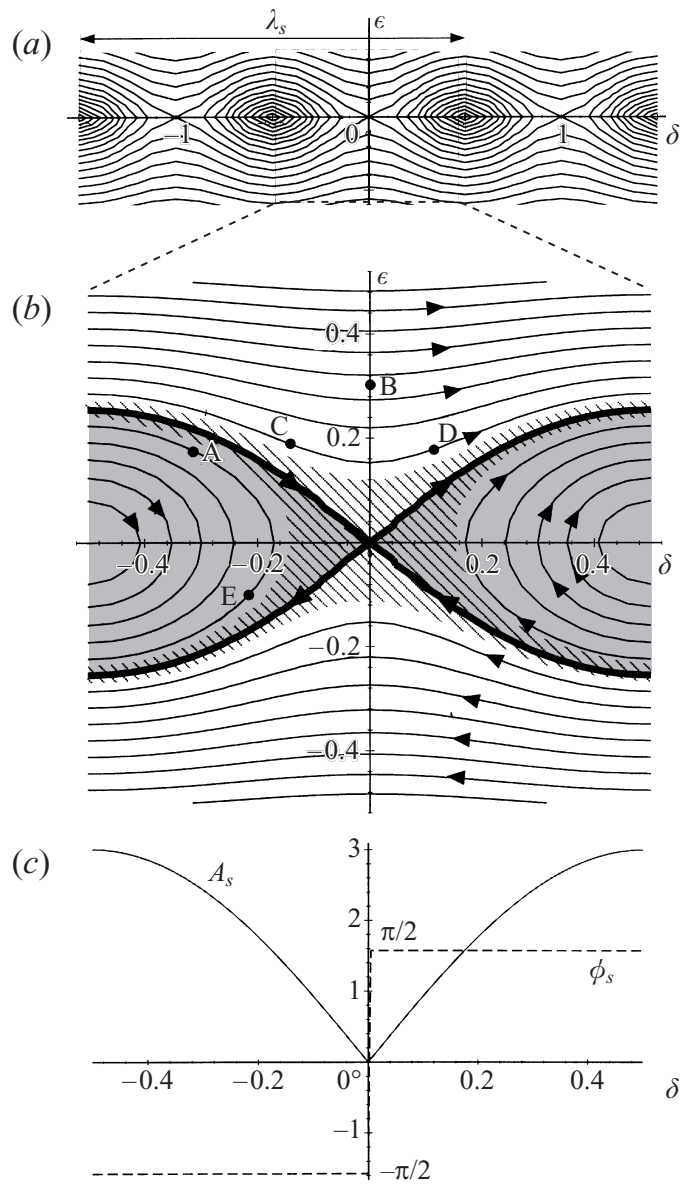

FiguRE 16. (a) The global and (b) detailed view of the phase trajectories of the system (5.7), $a=0.1$. (c) The subharmonic amplitude and the phase behaviour. The location of the stochastic layer is shown by a striped region.

of two lines of vortices with appropriate streamwise and lateral shifts (see figure 15):

$$
u(x, y ; \delta, \varepsilon, a)=\frac{1}{2}\left[u_{0}\left(\frac{x+\delta}{2}, \frac{y+\varepsilon}{2}, \frac{a}{2}\right)+u_{0}\left(\frac{x-\delta+\lambda_{f}}{2}, \frac{y-\varepsilon}{2}, \frac{a}{2}\right)\right],
$$

where $u_{0}(x, y, a)$ is defined by (5.2). A Fourier expansion of (5.10) gives

$$
\begin{aligned}
u(x, y ; \delta, \varepsilon, a)= & \frac{1}{2}\left[A_{0}\left(\frac{y+\varepsilon}{2}, \frac{a}{2}\right)+A_{0}\left(\frac{y-\varepsilon}{2}, \frac{a}{2}\right)\right] \\
& +A_{1}\left(\frac{y+\varepsilon}{2}, \frac{a}{2}\right) \cos \left(2 \pi \frac{x+\delta}{\lambda_{s}}\right) \\
& +A_{1}\left(\frac{y-\varepsilon}{2}, \frac{a}{2}\right) \cos \left(2 \pi \frac{x-\delta-\lambda_{f}}{\lambda_{s}}\right)+\cdots,
\end{aligned}
$$

with $A_{0}(y, a)=\int_{0}^{1} u_{0}(x, y, a) \mathrm{d} x=2 \pi y /\left(a^{2}+y^{2}\right)^{1 / 2}$ and $A_{1}(y, a)=\int_{0}^{1} u_{0}(x, y, a) \cos (2 \pi x)$ $\mathrm{d} x=2 \pi y /\left(a^{2}+y^{2}\right)^{1 / 2} \exp \left(-2 \pi\left(a^{2}+y^{2}\right)^{1 / 2}\right)$. The subharmonic term is found in the 
form $A_{s}(\delta, \varepsilon, a) \exp \left(\mathrm{i}\left(2 \pi x / \lambda_{s}+\phi_{s}(\delta)\right)\right)$ with

$$
\left.\begin{array}{rl}
A_{s}(\delta, \varepsilon, a) & =A(\varepsilon, a)\left|\sin \left(2 \pi \delta / \lambda_{s}\right)\right|, \\
\phi_{s}(\delta) & =\pi / 2 \operatorname{sign}\left(\sin \left(2 \pi \delta / \lambda_{s}\right)\right)
\end{array}\right\}
$$

whose behaviour versus $\delta$ for fixed $\varepsilon$ is plotted in figure 16(c).

The subharmonic mode has two stable points: $\phi_{1}=\pi / 2$ if $\sin (\pi \delta)>0$ and $\phi_{2}=-\pi / 2$ if $\sin (\pi \delta)<0$. For a phase-space location in the shaded region (e.g. point A of figure 16b) framed by a separatrix (shown as a thick dark line) the subharmonic phase $\phi_{s}$ remains constant and in this sense the region can be called a stable one. In the outer region, when the trajectory $(\delta(t), \varepsilon(t))$ crosses the line $\delta=0$ (point B of figure $16 b$, for example), the subharmonic phase $\phi_{s}$ experiences a $\pi$-jump while the amplitude $A_{s}$ drops to zero as shown in figure 16(c). The similarity between the model subharmonic behaviour shown in this figure and the experimental results highlighted in the inset region of figure 2 is readily apparent.

It is important to know that although the system is deterministic, it is very sensitive to the conditions in the neighbourhood of the separatrix. Any small variations of $\varepsilon$ and/or $\delta$ can make the system leave a stable region, giving rise to changes in $\phi_{s}$, since trajectories in the outer region result in $\pi$ phase jumps and subharmonic amplitude minima. Alternatively the system could return back to a stable region, where $\phi_{s}$ remains constant.

Note that the temporal behaviour of the system in the outer region can be described as a tearing and re-pairing process. The tearing phenomenon implies that the interaction within a pair of vortices becomes weak, the nascent vortex pair tears apart and the vortices start to behave as isolated ones. In contrast, the temporal behaviour in the inner region corresponds to vortex pairing.

\subsection{On the application of the model to the jet shear layer}

In the jet shear layer excited at low-amplitude an array of well-defined vortices exists for $70<x / \theta_{0}<200$. The fundamental wavelength $\lambda_{f}=U_{c} / f_{f} \approx 4 \mathrm{~mm}$ gives a rough estimate of the distance between vortices which, in turn, suggests that $M \approx 4-5$ spanwise two-dimensional vortices exist in this region at any given time. The theory which has been developed is based on an infinite number of vortices and cannot be applied directly to a finite line of vortices. However, let us consider any pair of vortices in this line with the corresponding $\varepsilon$ and $\delta$. The governing system for the dynamics of the pair is

$$
\frac{\mathrm{d} \varepsilon}{\mathrm{d} t}=\frac{\partial H}{\partial \delta}, \quad \frac{\mathrm{d} \delta}{\mathrm{d} t}=-\frac{\partial H}{\partial \varepsilon} .
$$

The Hamiltonian $H$ for the pair of vortices can be written as $H=H_{\infty}+\Delta H$, where the Hamiltonian $H_{\infty}$ corresponds to an infinite line of vortices, properly aligned according to (5.6) for a given $\varepsilon$ and $\delta$, and $\Delta H$ is the deviation of the Hamiltonian due to the difference between this ideal alignment of an infinite number of vortices and the real finite line of vortices. This difference consists of three parts: (i) the absence of vortices beyond the finite line of vortices, (ii) misalignment of vortices from a perfectly aligned case (as given by (5.6)) within the finite line and finally (iii) additional disturbances such as the influence from the vortical structures in the other jet shear layer. One can estimate that the absence of vortices outside of the finite line contributes $\sim 1 / M$ to $\Delta H$, where $M$ is the number of vortices in the finite line. From the fact that the subharmonic mode is well-defined and there are no other 
significant modes beside the fundamental and the subharmonic in this region of the flow, we conclude that the deviation in alignment of vortices from that given by (5.6) is small. So, the only difference between the dynamics of the pair of vortices in the finite line of vortices, equation (5.13), governed by $H$ and the dynamics of an infinite array of vortices, (5.7), governed by $H_{\infty}$, will be a small, general random force given by $(\partial(\Delta H) / \partial \delta,-\partial(\Delta H) / \partial \varepsilon)$. Thus, in order to account for the effects described above all we need to do is consider the dynamics of the system (5.7) under the influence of a small stochastic forcing.

In Rabinovich \& Trubetskov (1989) and Gaponov-Grekhov \& Rabinovich (1992) it is rigorously demonstrated that stochastic forcing will create local instabilities which will eventually destroy all phase trajectories passing through saddle point(s) to create a region of finite width with a stochastic behaviour. This region is termed a stochastic layer. Phase trajectories around other critical points like centres will remain topologically unchanged. In our case, the separatrix is the line connecting the saddle points (see figure 16) and thus the presence of a stochastic force will create a stochastic layer around it. This is shown as a striped region overlaying the separatrix in figure 16. The dynamics of the system within a stochastic layer is very complex and will not be discussed in detail here. The interested reader is referenced to Rabinovich \& Trubetskov (1989) for an extensive discussion. However, an especially important result is that in this layer there are no well-defined phase trajectories and the system can travel across it and in so doing move between inner and outer regions (i.e. between shaded and non-shaded regions of figure $16 b$ ). Nevertheless, the system still has two stable points $\phi_{1}$ and $\phi_{2}$. Every time its trajectory crosses the line $\delta=0$ it exhibits the $\pi$-jump in subharmonic phase and the subharmonic amplitude drops to zero. Therefore, the $\pi$-jump scenario will hold locally for the pair of vortices.

Consider a given pair of vortices 'emerging' at the $x / \theta_{0} \approx 70$ location. Depending on the initial conditions, a pair can find itself either in the stable inner region (e.g. point A) or in the outer region (e.g. point C) of figure 16(b). As any given pair of vortices evolves in time and moves downstream, which could, for example, correspond to either one of the paths $\mathrm{A}-\mathrm{E}$ or $\mathrm{C}-\mathrm{D}$ in figure $16(b)$, the subharmonic phase $\phi_{s}$ would be either $\phi_{1}=-\pi / 2(\delta<0$, e.g. point $\mathrm{E})$ or $\phi_{1}=\pi / 2(\delta>0$, e.g. point $\mathrm{D})$. If a trajectory starts in the shaded region of figure $16(b)$, it will stay in the stable region with $\phi_{s}=-\pi / 2$. Otherwise it will eventually cross the line $\delta=0$ and the phase will become $\phi_{s}=\pi / 2$. For points in the shaded region but very near the stochastic layer, perturbations in $\varepsilon$ and/or $\delta$ can make the system leave a stable region thereby giving rise to changes in $\phi_{s}$.

The $\delta$-parameter combines both the subharmonic streamwise perturbation and the inherent variation of the fundamental wavelength (frequency), while the $\varepsilon$-parameter contains the cumulative effect of the subharmonic lateral perturbation both upstream and downstream of location $x$. Let us suppose that the initial probability distribution of $\delta$ is Gaussian-like with zero mean. From figure 17 it is easy to see that for $\varepsilon=0$ both stable points will be equally probable with $P_{1}=P_{2}$. Physically this situation corresponds most closely to natural or very low-level subharmonic excitation. Note, for instance, that both phase plateaux in the experimental results shown in figure 2 are equally visited in time. However, figure 17 also shows that for larger values of $\varepsilon>0$ corresponding to cases with higher local levels of the subharmonic perturbation, the probability $P_{2}$ of the system evolving toward the subharmonic phase $\phi_{s}=\phi_{2}$ is greater than the probability $P_{1}$ for the subharmonic phase to be $\phi_{s}=\phi_{1}$. This behaviour can be observed by comparing experimental results like those shown in figures 2 and 12. Figure 12, which corresponds to a higher local level of subharmonic 


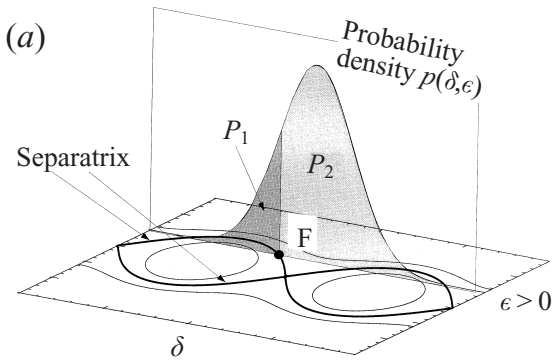

(b)

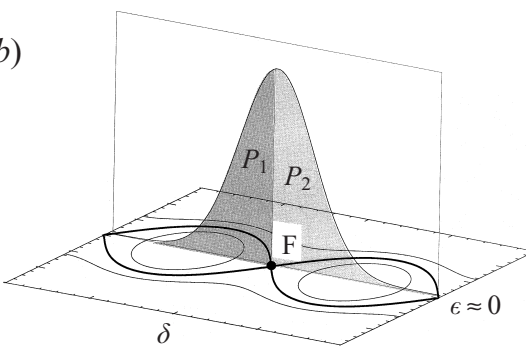

FIGURE 17. Schematic of relative probabilities for the subharmonic phase: (a) $\varepsilon>0$ and $(b) \varepsilon \approx 0$.

perturbation (due to a higher $u_{f}^{\prime}$ ), shows that one of the values of subharmonic phase is more probable than the other, with one value visited $8 \%$ of the time and the other $92 \%$.

To show more directly how the model compares with experimental results, we introduce a small-amplitude subharmonic perturbation in the $y$-direction as $y_{j}=$ $\varepsilon_{0} \cos \left(2 \pi j / \lambda_{s}\right)$ and a random deviation from an equally spaced streamwise vortex distribution as $x_{j}=j+\delta_{0} N(0,1)$. Here $N(0,1)$ denotes the normal probability distribution with mean value 0 and standard deviation 1 while $\varepsilon_{0}$ and $\delta_{0}$ are small constant parameters. From the experiments involving a moderate local subharmonic perturbation level it was found that $0.07 \leqslant a / \lambda_{f} \leqslant 0.1$ and $0.05 \leqslant \varepsilon / \lambda_{f} \leqslant 0.1$. Using a Runge-Kutta integration method the system (5.3) with 1500 vortices for $\varepsilon_{0}=\delta_{0}=0.05$ and $a=0.1$ was numerically solved to find the resulting locations of vortices $\left(x_{j}, y_{j}\right)$ in time $t$. After restoring the resulting continuous velocity field, a Morlet wavelet transformation was applied just as in the experiments. The resulting spatial evolution of both the fundamental and subharmonic modes for $t=0.1 \mathrm{~s}$ was obtained and results for the subharmonic are presented in figure 18. It should be noted that the effect of core radius was investigated and the resulting phase trajectories showed that the qualitative system dynamics were not sensitive to the assumed $a$ value.

Comparison of figure 18 with experimental data presented in figure 12 shows very good agreement regarding the essential aspects of the unsteady shear layer behaviour. In comparing figures 18 and 12 recall that the spatial behaviour of the model corresponds to temporal evolution in the real experiment. Although not presented it should be noted that for both the model and experiment the fundamental exhibits low-level random variation about statistically steady mean values in both amplitude and phase. In contrast, the subharmonic phase for both experiment and model shows a similar unsteady behaviour characterized by sudden $\pi$-jumps. During these jumps 

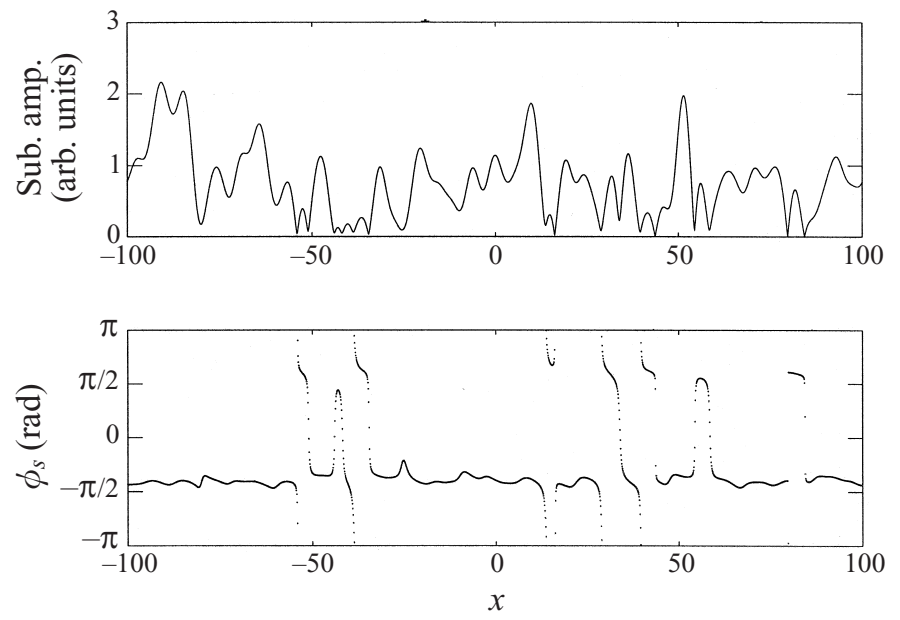

Figure 18. Computed spatial behaviour of the subharmonic mode after 0.1 s. $\varepsilon_{0}=\delta_{0}=0.05$.
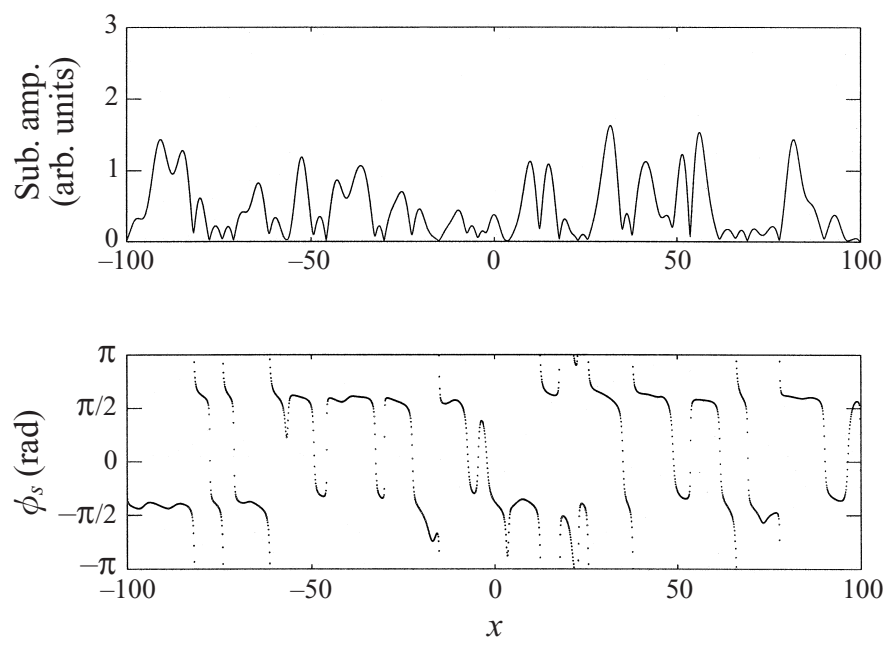

Figure 19. Computed spatial behaviour of the the subharmonic mode after $0.1 \mathrm{~s}$. $\varepsilon_{0}=0.0001, \delta_{0}=0.05$.

the subharmonic amplitude approaches zero in both experiment and model. The experiment shows a much greater probability for one value of subharmonic phase than the other possible value and this is also in agreement with the model result.

Consider next the case of very small local subharmonic perturbation corresponding to $\varepsilon \approx 0$. Computational results are presented in figure 19 for $\varepsilon_{0}=0.0001, \delta_{0}=0.05$, $t=0.1 \mathrm{~s}$. Now the system is observed to visit both subharmonic phase values equally in time which is consistent with the experimental results presented in figure 2.

The computational results shown in figures 18 and 19 are consistent with the discussion surrounding figure 17 and demonstrate how the subharmonic phase can spend more time at one phase value than another for moderate local subharmonic excitation.

It is important to note the difference between temporal $\pi$-jumps in phase for the natural subharmonic mode and the $\pi$-shifts in average effective $F-S$ phase angle 
between pairing and tearing regimes as observed in the bimodal forcing experiments (figures 6 and 7). Sustained vortex tearing is not observed in experiments involving the naturally occurring subharmonic. Even in the experiments involving bimodal excitation, in which local subharmonic suppression is achieved by proper selection of $\phi_{i n}$, it was found that such regions are characterized by a high level of temporal phase variation. This is because the vortex tearing regime is associated with an unstable saddle point in phase space. Consequently trajectories will not remain there for the natural subharmonic and even with artificial excitation of the subharmonic it is difficult to keep the phase trajectory near the saddle point. In fact, application of the wavelet transform to regions of the flow characterized by subharmonic suppression in the bimodal forcing experiments showed temporal subharmonic amplitude and phase behaviour which is similar in character to that observed in experiments involving the natural subharmonic. In contrast, regions of strong subharmonic growth are found to be associated with little temporal phase variation, a consequence of the stability inherent in the two fixed points. In the bimodal forcing experiments, the streamwise variation in the local effective $\mathrm{F}-\mathrm{S}$ phase for $\phi_{\text {in }}$ corresponding to vortex pairing and tearing are separated by $\pi$ radians for $x$-stations where the subharmonic is suppressed. This is consistent with the Hamiltonian model. When the system is forced to be in an unstable tearing regime the trajectory lies in the neighbourhood of the saddle point in figure $16(b)$. This corresponds to the case of $\phi_{s}=0$ and $A_{s} \approx 0$ (see figure 16c), while the pairing scenario corresponds to $\phi_{s}= \pm \pi / 2$ and peak amplitude $A_{s}$ (i.e. the centres in figure 16a). The difference in local effective $\mathrm{F}-\mathrm{S}$ phase angle between cases is $\left[\phi_{\text {eff }}\right]=\left[\phi_{f}\right]-2\left[\phi_{s}\right]$, where [·] denotes the difference in values between the pairing and tearing regimes. As $\left[\phi_{f}\right]=0,\left[\phi_{\text {eff }}\right]=-2\left[\phi_{s}\right]=-2( \pm \pi / 2)=\mp \pi$ as indicated in the experimental results shown in figures 5, 6 and 7 .

The model reflects all essential features of the vortex interactions within the shear layer: the unsteady subharmonic amplitude variation and the existence of two pseudostable phase points with sudden $\pi$-jumps between them. The phase excursions and the amplitude drops occur at the same time.

The dissipative effect of viscosity will change centre points into stable foci which is equivalent to the eventual merging of two pairing vortices into one. However, it is important to note that viscosity will leave the saddle point unchanged so that the $\pi$-jump scenario will still hold in the presence of viscosity.

Of course, since the model is inherently two-dimensional it will fail to apply at streamwise locations well downstream of vortex pairing where the initially spanwise coherent vortex filaments become three-dimensional and mixing transition has occurred.

\section{Summary}

In experiments utilizing excitation of only the fundamental mode over the range $0.002 \% \leqslant u_{f}^{\prime} \leqslant 0.1 \%$, the naturally developing subharmonic exhibits an interesting temporal amplitude and phase variation that has not been previously reported. This takes the form of intermittent $\pi$-shifts in subharmonic phase between two fixed phase values. These phase jumps are observed to be highly correlated with the local drops to a near zero value of the subharmonic amplitude. In contrast, the fundamental amplitude and phase show no such behaviour, exhibiting only low levels of temporal modulation. The temporal phase behaviour of the subharmonic has the effect of intermittently disrupting the phase lock with the fundamental. Similar behaviour is 
encountered in bimodal forcing experiments for those initial effective phase angles $\phi_{i n}$ that are found to give rise to subharmonic mode suppression.

A Hamiltonian formulation of the problem is shown to provide remarkable agreement with the experimental results. All the essential aspects of the temporal amplitude and phase behaviour of the subharmonic are reproduced by the model including amplitude-dependent effects. The model is also shown to provide a dynamical systems based explanation for time-average amplitude and effective phase behaviour reported in previous investigations. Intermittent subharmonic phase shifts and subharmonic amplitude minima observed in the wavelet transformation correspond to isolated vortex tearing events. A key element in giving rise to the observed dynamic behaviour is the existence of a stochastic layer in which phase-space trajectories can travel from one stable pairing regime to another. In the real flow the stochastic layer is associated with perturbations to a local pair of vortices from both upstream and downstream vortical structures as well as the shear layer on the other side of the jet potential core.

The $\pi$-jump scenario for subharmonic phase is the rule in experiments involving low-amplitude forcing of the fundamental and the natural development of the subharmonic. The model demonstrates that this is because the vortex tearing regime is associated with an unstable saddle point in phase space. As a consequence, system trajectories will not remain there for the natural subharmonic, and even with artificial excitation of the subharmonic instability in bimodal forcing experiments, it is difficult to keep the phase trajectory near the saddle point (even if the forcing amplitudes are quite large). As a consequence there is a very narrow range of $\phi_{i n}$ which is capable of giving rise to subharmonic mode suppression. Application of the wavelet transform to regions of the flow characterized by subharmonic mode suppression in the bimodal forcing experiments shows temporal subharmonic amplitude and phase behaviour which is similar in character to that observed in experiments involving the natural subharmonic. In contrast, regions of strong subharmonic growth are found to be associated with little temporal phase variation, a consequence of the stability inherent in the two fixed points.

The model is able to explain why there is a higher probability for the subharmonic phase to stay near one of the stable phase points with increases in initial excitation amplitude. In fact, numerical simulations based on the model show a very good agreement with the corresponding wavelet-based experimental data in this regard.

In addition, as described in $\S 5.2$, the dynamical systems model provides a sound explanation for the $\pi$-shift in time-average effective phase angle $\phi_{\text {eff }}$ that occurs in the bimodal forcing experiments at a fixed streamwise location between vortex pairing and tearing regimes.

\section{Appendix}

Consider the sum

$$
\begin{aligned}
H & =-\frac{1}{2} \sum_{n=-\infty}^{n=+\infty} \ln \left\{\left[1+\frac{4\left(\varepsilon^{2}+\delta^{2}\right)}{a^{2}+(2 n+1)^{2}}\right]^{2}-\left[\frac{4 \delta(2 n+1)}{a^{2}+(2 n+1)^{2}}\right]^{2}\right\} \\
& =-\frac{1}{2} \sum_{n=-\infty}^{n=+\infty} \ln \frac{Q\left(\frac{1}{2}(2 n+1)\right)}{\left[a^{2}+(2 n+1)^{2}\right]^{2}},
\end{aligned}
$$

where

$$
Q(j)=16\left[\tilde{a}^{2}+j^{2}+\left(\varepsilon^{2}+\delta^{2}\right)\right]^{2}-4 \delta^{2} j^{2}, \quad \tilde{a}=a / 2 .
$$


Consider

$$
\frac{\partial H}{\partial \varepsilon}=-\frac{1}{2} \sum_{n=-\infty}^{n=+\infty} \frac{\frac{\partial Q\left(\frac{1}{2}(2 n+1)\right)}{\partial \varepsilon}}{Q\left(\frac{1}{2}(2 n+1)\right)}=-\frac{1}{2} \sum_{n=-\infty}^{n=+\infty} f\left(\frac{(2 n+1)}{2}\right) .
$$

Use the Mittag-Leffler's formula,

$$
\sum_{n=-\infty}^{n=+\infty} f\left(\frac{1}{2}(2 n+1)\right)=\pi \sum_{\text {all poles of } f(z)} \operatorname{Res}\{\tan \pi z f(z)\} .
$$

Poles of $f(z)$ correspond to zeros of the polynomial $Q(z)$. $Q(z)$ has four zeros: $z_{1,2,3,4}= \pm \delta \pm \mathrm{i} \sqrt{\tilde{a}^{2}+\varepsilon^{2}}$. For the $z$ 's

$$
\begin{gathered}
\tan \pi z_{1,2,3,4}=\frac{1}{2} \frac{ \pm \sin (2 \pi \delta) \pm \mathrm{i} \sinh \sqrt{\tilde{a}^{2}+\varepsilon^{2}}}{\cos ^{2} \pi \delta+\sinh ^{2}\left(\pi \sqrt{\tilde{a}^{2}+\varepsilon^{2}}\right)}, \\
\operatorname{Res} f\left(z_{1,3}\right)=-\operatorname{Res} f\left(z_{2,4}\right)=-\mathrm{i} \frac{\varepsilon}{\sqrt{\tilde{a}^{2}+\varepsilon^{2}}} .
\end{gathered}
$$

Finally

$$
\frac{\partial H}{\partial \varepsilon}=-\frac{\pi \varepsilon}{\sqrt{\tilde{a}^{2}+\varepsilon^{2}}} \frac{\sinh \left(2 \pi \sqrt{\tilde{a}^{2}+\varepsilon^{2}}\right)}{\cos ^{2}(\pi \delta)+\sinh ^{2}\left(\pi \sqrt{\tilde{a}^{2}+\varepsilon^{2}}\right)} .
$$

Similarly,

$$
\frac{\partial H}{\partial \delta}=-\frac{1}{2} \sum_{n=-\infty}^{n=+\infty} \frac{\frac{\partial Q\left(\frac{1}{2}(2 n+1)\right)}{\partial \delta}}{Q\left(\frac{1}{2}(2 n+1)\right)}=-\frac{1}{2} \sum_{n=-\infty}^{n=+\infty} g\left(\frac{(2 n+1)}{2}\right)
$$

with the final result

$$
\frac{\partial H}{\partial \delta}=\pi \frac{\sin (2 \pi \delta)}{\cos ^{2}(\pi \delta)+\sinh ^{2}\left(\pi \sqrt{\tilde{a}^{2}+\varepsilon^{2}}\right)} .
$$

Reconstruction of $H$ from $\partial H / \partial \varepsilon$ and $\partial H / \partial \delta$ gives

$$
H(\delta, \varepsilon)=-\frac{1}{2} \log \left[\cos ^{2}(\pi \delta)+\sinh ^{2}\left(\pi \sqrt{(a / 2)^{2}+\varepsilon^{2}}\right)\right] .
$$

\section{REFERENCES}

Arbey, H. \& Ffowcs Williams, J. E. 1984 Active cancellation of pure tones in an excited jet. J. Fluid Mech. 149, 445-454.

Batchelor, G. K. 1967 An Introduction to Fluid Dynamics. Cambridge University Press.

Bendat, J. S. \& Piersol, A. G. 1986 Random Data: Analysis and Measurement Procedures, 2nd Edn. John Wiley.

Betyayev, S. K., Gaifullin, A. M. \& Gordeyev, S. V. 1994 The point-circle vortex. J. Appl. Maths Mech. 58(4), 749-754.

Corke, T. C., Shakib, F. \& Nagib, H. 1991 Mode selection and resonant phase locking in unstable axisymmetric jets. J. Fluid Mech. 223, 253-311.

Daubechies, I. 1992 Ten Lectures on Wavelets. Society for Industrial and Applied Mathematics.

Drazin, P. G. \& ReID, W. H. 1983 Hydrodynamic Stability. Cambridge University Press.

FARGE, M. 1992 Wavelet transforms and their applications to turbulence. Ann. Rev. Fluid Mech. 24 395-457. 
Farge, M., Kevlahan, N., Perrier, V. \& Goirand, E. 1996 Wavelets and turbulence. Proc. IEeE 84(4), 639-669.

Gaponov-Grekhov, A. V. \& Rabinovich, M. I. 1992 Nonlinearities in Action: Oscillations, Chaos, Order, Fractals. Springer.

HajJ, M. R., Miksad, R. W. \& Powers, E. J. 1992 Subharmonic growth by parametric resonance. J. Fluid Mech. 236, 385-413.

HajJ, M. R., Miksad, R. W. \& Powers, E. J. 1993 Fundamental-subharmonic interaction: effect of phase relation. J. Fluid Mech. 256, 403-426.

Ho, C. M. \& Huang, L. S. 1982 Subharmonics and vortex merging in mixing layers. J. Fluid Mech. 119, 443-473.

Hunng, L. S. \& Ho, C. M. 1990 Small-scale transition in a plane mixing layer. J. Fluid Mech. 210, 475-500.

Husain, H. \& Hussain, F. 1995 Experiments on subharmonic resonance in a shear layer. J. Fluid Mech. 304, 343-372.

KAISER, G. 1994 A Friendly Guide to Wavelets. Birkhauser.

Kelly, R. E. 1967 On the stability of an inviscid shear layer which is periodic in space and time. J. Fluid Mech. 31, 789-99.

Kim, Y. C., Khadra, L. \& Powers, E. J. 1980 Wave modulation in a nonlinear dispersive medium. Phys. Fluids, 23, 2250-2257.

LAMB, H. 1945 Hydrodynamics. Dover.

LEWALLE, J. 1994 Wavelet analysis of experimental data: some methods and the underlying physics. AIAA Paper 94-2281.

MANKBADI, R. R. 1985 On the interaction between fundamental and subharmonic instability waves in a turbulent round jet. J. Fluid Mech. 160, 385-419.

MankBADI, R. R. 1986 The effect of phase difference on the spreading rate of a jet. AIAA J. 24, 1941-1948.

Mankbadi, R. R. 1994 Transition, Turbulence, and Noise: Theory and Applications for Scientists and Engineers. Kluwer.

Monkewitz, P. A. 1988 Subharmonic resonance, pairing and shredding in the mixing layer. J. Fluid Mech. 188, 223-252.

Moore, D. W. \& Saffman, P. G. 1975 The density of organized vortices in a turbulent mixing layer. J. Fluid Mech. 69, 465-473.

Rabinovich, M. I. \& Trubetskov, D. I. 1989 Oscillations and Waves in Linear and Nonlinear Systems. Kluwer.

Rajaee, M. \& Karlsson, S. K. F. 1992 On the Fourier space decomposition of free shear flow measurements and mode degeneration in the pairing process. Phys. Fluids A 4, 321-339.

Thomas, F. O. \& CHU, H. C. 1991 Experimental investigation of the nonlinear spectral dynamics of planar jet transition. Phys. Fluids 3, 1544-1559.

Thomas, F. O. \& CHU, H. C. $1993 a$ Nonlinear wave coupling and subharmonic resonance in planar jet shear layer transition. Phys. Fluids A 5, 630-646.

Thomas, F. O. \& CHU, H. C. $1993 b$ Experiments on the nonlinear stages of excited jet shear layer transition. Exps. Fluids 14, 451-467.

YANG, Z. \& KARLSSON, S. K. F. 1991 Evolution of coherent structures in a plane shear layer. Phys. Fluids A 3, 2207-2219.

Zhang, Y. Q., Ho, C. M. \& Monkewitz, P. 1983 The mixing layer forced by fundamental and subharmonic in laminar-turbulent transition. IUTAM Symp, Novosibirsk, USSR (ed. V. V. Kozlov), pp. 385-395. Springer. 